\title{
A detailed spectral and morphological study of the gamma-ray supernova remnant RX J1713.7-3946 with HESS
}

F. Aharonian ${ }^{1}$, A. G. Akhperjanian ${ }^{2}$, A. R. Bazer-Bachi ${ }^{3}$, M. Beilicke ${ }^{4}$, W. Benbow ${ }^{1}$, D. Berge ${ }^{1}$, K. Bernlöhr ${ }^{1,5}$, C. Boisson ${ }^{6}$, O. Bolz ${ }^{1}$, V. Borrel ${ }^{3}$, I. Braun ${ }^{1}$, F. Breitling, ${ }^{5}$, A. M. Brown ${ }^{7}$, P. M. Chadwick ${ }^{7}$, L.-M. Chounet ${ }^{8}$, R. Cornils ${ }^{4}$, L. Costamante ${ }^{1,20}$, B. Degrange ${ }^{8}$, H. J. Dickinson ${ }^{7}$, A. Djannati-Ataï ${ }^{9}$, L. O'C. Drury ${ }^{10}$, G. Dubus ${ }^{8}$, D. Emmanoulopoulos ${ }^{11}$, P. Espigat ${ }^{9}$, F. Feinstein ${ }^{12}$, G. Fontaine ${ }^{8}$, Y. Fuchs ${ }^{13}$, S. Funk ${ }^{1}$, Y. A. Gallant ${ }^{12}$, B. Giebels ${ }^{8}$, J. F. Glicenstein ${ }^{14}$, P. Goret ${ }^{14}$, C. Hadjichristidis ${ }^{7}$, D. Hauser ${ }^{1}$, M. Hauser ${ }^{11}$, G. Heinzelmann ${ }^{4}$, G. Henri ${ }^{13}$, G. Hermann ${ }^{1}$, J. A. Hinton ${ }^{1,11}$, W. Hofmann ${ }^{1}$, M. Holleran ${ }^{15}$, D. Horns ${ }^{1}$, A. Jacholkowska ${ }^{12}$, O. C. de Jager ${ }^{15}$, B. Khélifi ${ }^{1}$, S. Klages ${ }^{1}$, Nu. Komin ${ }^{5}$, A. Konopelko ${ }^{5}$, I. J. Latham ${ }^{7}$, R. Le Gallou ${ }^{7}$, A. Lemière ${ }^{9}$,

M. Lemoine-Goumard ${ }^{8}$, T. Lohse ${ }^{5}$, J. M. Martin ${ }^{6}$, O. Martineau-Huynh ${ }^{16}$, A. Marcowith ${ }^{3}$, C. Masterson ${ }^{1,20}$, T. J. L. McComb ${ }^{7}$, M. de Naurois ${ }^{16}$, D. Nedbal ${ }^{17}$, S. J. Nolan ${ }^{7}$, A. Noutsos ${ }^{7}$, K. J. Orford ${ }^{7}$, J. L. Osborne ${ }^{7}$, M. Ouchrif ${ }^{16,20}$, M. Panter ${ }^{1}$, G. Pelletier ${ }^{13}$, S. Pita ${ }^{9}$, G. Pühlhofer ${ }^{11}$, M. Punch ${ }^{9}$, B. C. Raubenheimer ${ }^{15}$, M. Raue ${ }^{4}$, S. M. Rayner ${ }^{7}$, A. Reimer ${ }^{18}$, O. Reimer ${ }^{18}$, J. Ripken ${ }^{4}$, L. Rob ${ }^{17}$, L. Rolland ${ }^{16}$, G. Rowell ${ }^{1}$, V. Sahakian ${ }^{2}$, L. Saugé ${ }^{13}$, S. Schlenker ${ }^{5}$, R. Schlickeiser ${ }^{18}$, C. Schuster ${ }^{18}$, U. Schwanke ${ }^{5}$, M. Siewert ${ }^{18}$, H. Sol $^{6}$, D. Spangler ${ }^{7}$, R. Steenkamp ${ }^{19}$, C. Stegmann ${ }^{5}$, G. Superina ${ }^{8}$, J.-P. Tavernet ${ }^{16}$, R. Terrier ${ }^{9}$, C. G. Théoret ${ }^{9}$, M. Tluczykont ${ }^{8,20}$, C. van Eldik ${ }^{1}$, G. Vasileiadis ${ }^{12}$, C. Venter ${ }^{15}$, P. Vincent ${ }^{16}$, H. J. Völk ${ }^{1}$, and S. J. Wagner ${ }^{11}$

(Affiliations can be found after the references)

Received 29 September 2005 / Accepted 12 November 2005

ABSTRACT

Aims. We present results from deep observations of the Galactic shell-type supernova remnant (SNR) RX J1713.7-3946 (also known as G347.3-0.5) conducted with the complete HESS array in 2004.

Methods. Detailed morphological and spatially resolved spectral studies reveal the very-high-energy (VHE - Energies $E>100 \mathrm{GeV}$ ) gamma-ray aspects of this object with unprecedented precision. Since this is the first in-depth analysis of an extended VHE gamma-ray source, we present a thorough discussion of our methodology and investigations of possible sources of systematic errors.

Results. Gamma rays are detected throughout the whole SNR. The emission is found to resemble a shell structure with increased fluxes from the western and northwestern parts. The differential gamma-ray spectrum of the whole SNR is measured over more than two orders of magnitude, from $190 \mathrm{GeV}$ to $40 \mathrm{TeV}$, and is rather hard with indications for a deviation from a pure power law at high energies. Spectra have also been determined for spatially separated regions of RX J1713.7-3946. The flux values vary by more than a factor of two, but no significant change in spectral shape is found. There is a striking correlation between the X-ray and the gamma-ray image. Radial profiles in both wavelength regimes reveal the same shape almost everywhere in the region of the SNR.

Conclusions. The VHE gamma-ray emission of RX J1713.7-3946 is phenomenologically discussed for two scenarios, one where the gamma rays are produced by VHE electrons via Inverse Compton scattering and one where the gamma rays are due to neutral pion decay from proton-proton interactions. In conjunction with multi-wavelength considerations, the latter case is favoured. However, no decisive conclusions can yet be drawn regarding the parent particle population dominantly responsible for the gamma-ray emission from RX J1713.7-3946.

Key words. acceleration of particles - ISM: cosmic rays - gamma rays: observations - ISM: supernova remnants ISM: individual objects: RX J1713.7-3946 (G347.3-0.5)

\section{Introduction}

It is commonly believed that the only sources capable of supplying enough energy output to power the flux of Galactic cosmic rays are supernova explosions (e.g., Ginzburg \& Syrovatskii 1964). At the present time there are two main arguments for this hypothesis: firstly, estimates of the power required to sustain the observed nuclear Galactic cosmic-ray population show that about $10 \%$ of the mechanical energy released by the population of Galactic supernovae would suffice, or, in other words, that supernova remnants could be the sources of the Galactic cosmic rays if the average acceleration efficiency in a remnant is about $10 \%$. Secondly, a rather well developed theoretical framework for the acceleration 
mechanism, diffusive shock acceleration (for reviews see e.g., Blandford \& Eichler 1987; Jones \& Ellison 1991; Malkov \& O'C Drury 2001), exists and it indeed predicts acceleration efficiencies in excess of $10 \%$.

The best way of proving unequivocally the existence of very-high-energy (VHE) particles, electrons or hadrons, in the shells of supernova remnants (SNRs) is the detection of VHE (about $100 \mathrm{GeV}$ up to a few tens of $\mathrm{TeV}$ ) gamma rays produced either via Inverse Compton (IC) scattering of VHE electrons off ambient photons or in interactions of nucleonic cosmic rays with ambient matter. As was argued already in Drury et al. (1994), a system of imaging atmospheric Cherenkov telescopes with a large field of view provides the most powerful measurement technique for extended nearby SNRs at these very high energies. One should note that there exist two other experimental approaches to trace VHE cosmic rays, the detection of X-rays, which suggests the presence of VHE electrons (Koyama et al. 1995), and of high-energy neutrinos, which probe exclusively nuclear particles.

A prime candidate for gamma-ray observations is the SNR RX J1713.7-3946, in particular because of its close association with dense molecular clouds along the line of sight (Fukui et al. 2003; Moriguchi et al. 2005), which might suggest a scenario of a supernova shell overtaking dense molecular clouds, leading to a detectable VHE gamma-ray signal from hadronic interactions, as described in Aharonian et al. (1994). RX J1713.7-3946, situated in the Galactic plane, constellation Scorpius, was discovered in soft X-rays in 1996 in the ROSAT all-sky survey (Pfeffermann \& Aschenbach 1996). It is roughly $70^{\prime}$ in diameter and exhibits bright X-ray emission dominantly from its western shell. ASCA observations revealed that the $\mathrm{X}$-ray emission is a pure non-thermal continuum without detectable line emission (Koyama et al. 1997; Slane et al. 1999). $\mathrm{X}$-ray observations have also been conducted with Chandra and XMM with their superior angular resolution. Chandra observed a small region in the bright northwestern part of the SNR (Uchiyama et al. 2003; Lazendic et al. 2004). Despite distinct brightness variations within this small field, the corresponding X-ray spectra were all found to be well described by power-law models with similar absorbing column densities and photon indices, albeit with rather large statistical uncertainties. XMM covered the remnant almost completely in five pointings (Cassam-Chenaï et al. 2004; Hiraga et al. 2005). Also on this much larger scale, a highly inhomogeneous and complex morphology was found in the western part of the SNR with two narrow rims resembling a double-shell structure running from north to south. The (non-thermal) X-ray spectra, when fit with a power law, exhibit strong variations in photon index across the remnant $(1.8<\Gamma<2.6)$ and the hydrogen column density $N_{\mathrm{H}}$ was found to vary significantly $\left(0.4 \times 10^{22} \mathrm{~cm}^{-2} \leq\right.$ $N_{\mathrm{H}} \leq 1.1 \times 10^{22} \mathrm{~cm}^{-2}$ ). The spectra of the central and the western parts differ clearly at low energies, possibly indicating an increase in column density of $\Delta N_{\mathrm{H}} \approx 0.4 \times 10^{22} \mathrm{~cm}^{-2}$ towards the west. Furthermore, a positive correlation between $\mathrm{X}$-ray brightness and absorption was interpreted as being due to the shock front of RX J1713.7-3946 impacting a molecular cloud in the west which was assumed to be responsible for the absorption. Further support for this scenario is lent by CO line emission observations with the NANTEN telescope (Fukui et al. 2003; Moriguchi et al. 2005), which suggest that the SNR is interacting with molecular clouds in this region at a distance of $1 \mathrm{kpc}$ from the Solar System. The non-thermal X-ray emission is possibly associated with interactions between the cloud and the western part of the SNR shell.

Age and distance of the SNR are under debate and have been revised quite a few times. Initially, Koyama et al. (1997) had derived a distance of $1 \mathrm{kpc}$ and correspondingly an age of about 1000 years from the column density towards the source as estimated from ASCA X-ray observations. Slane et al. (1999) on the other hand have derived a larger distance of $6 \mathrm{kpc}$ (corresponding to an age of about 10000 years) based on the possible association of RX J1713.7-3946 with a molecular cloud in this region and the $\mathrm{H}$ II region G347.6+0.2 to its northwest. Both the latest XMM and NANTEN findings are consistent with the remnant being closer, at $1 \mathrm{kpc}$, which might support the hypothesis of Wang et al. (1997), that RX J1713.7-3946 is the remnant of a AD393 guest star which, according to historical records, appeared in the tail of constellation Scorpius, close to the actual position of RX J1713.7-3946. The high surface brightness of this object, both in VHE gamma rays and non-thermal X-rays, suggests that it is close to the evolutionary phase where the shocks are most powerful. While hardly a conclusive argument, this implies that the remnant is observed at the sweep-up time when the ejecta are interacting with approximately their own mass of swept-up ambient material and the energy flux through the shocks (both forward and reverse) peaks. Normally this would be at an age of a few hundred to a thousand years, which indeed supports the closer distance estimate.

The radio emission of RX J1713.7-3946 is very faint (Lazendic et al. 2004) which puts it into a peculiar class of shell-type SNRs with dominantly non-thermal X-ray and only very faint radio emission. The only other known object of this type is RX J0852.0-4622 (G266.2-1.2) (Aschenbach 1998; Slane et al. 2001).

RX J1713.7-3946 was detected in VHE gamma rays by the CANGAROO collaboration in 1998 (Muraishi et al. 2000) and re-observed by CANGAROO-II in 2000 and 2001 (Enomoto et al. 2002). Recently HESS, a new array of imaging atmospheric Cherenkov telescopes operating in Namibia, has confirmed the detection (Aharonian et al. 2004b). This was the first independent confirmation of VHE gamma-ray emission from an SNR shell. Furthermore, the HESS measurement provided the first ever resolved gamma-ray image at very high energies. The complex morphology of RX J1713.7-3946 was clearly unraveled. Together with the HESS detection of RX J0852.0-4622 (Aharonian et al. 2005a) there are currently two spatially resolved VHE gamma-ray SNRs with a shelllike structure which agrees well with that seen in X-rays. These two objects may well be the brightest SNRs in the VHE gamma-ray domain in the whole sky; anything equally bright in the Northern sky would have been clearly seen in the Milagro survey (Atkins et al. 2004), and the HESS Galactic plane survey (Aharonian et al. 2006) reveals no SNRs brighter than RX J1713.7-3946 or RX J0852.0-4622 in the region covered. 
The interpretation of the gamma-ray emission mechanisms for RX J1713.7-3946 has been the subject of debate. From their flux level, the CANGAROO collaboration interpreted in Muraishi et al. (2000) the gamma rays as IC emission, whereas in Enomoto et al. (2002), after re-observations with CANGAROO-II, neutral pion decay was put forward as an explanation instead. The proposed model was then heavily disputed by Reimer \& Pohl (2002) and Butt et al. (2002) because of its conflict in the $\mathrm{GeV}$ regime with the flux of the nearby EGRET source 3EG 1714-3857 (Hartman et al. 1999). Further attempts to model the broadband spectrum of RX J1713.7-3946 were undertaken (e.g., Ellison et al. 2001; Uchiyama et al. 2003; Pannuti et al. 2003; Lazendic et al. 2004). However, they did not result in unequivocal conclusions concerning the acceleration mechanisms of the highest-energy particles or the origin of the VHE gamma rays from this source.

Here we report on follow-up observations of RX J1713.73946 with the complete HESS telescope array, conducted in 2004. The large field of view together with the high sensitivity of the system enable us to undertake for the first time detailed morphological studies in VHE gamma rays and measure spectral parameters in different regions of the SNR.

The paper is organised as follows: in Sect. 2 we present the data set and illustrate the performance of HESS for observations of extended gamma-ray sources. We explain in detail the analysis methods applied here in order to extract images and spectra. In Sect. 3 the results of spectral and morphological studies are presented along with systematic tests that have been performed in order to assure the validity of the analysis. Section 4 presents multi-wavelength data of RX J1713.7-3946 and its surroundings aiming at putting our measurement at $\mathrm{TeV}$ energies into the context of the available data as preparation for a broadband modelling of the spectral energy distribution (SED) in Sect. 5. We discuss two scenarios for the generation of VHE gamma rays, a purely electronic and a purely hadronic one. The results are finally summarised in Sect. 6 .

\section{Data processing}

\subsection{HESS observations}

Observations of RX J1713.7-3946 were conducted between April and July 2004 with the High Energy Stereoscopic System (HESS), a system of four imaging atmospheric Cherenkov telescopes (Hinton 2004; Hofmann 2003) situated in the Khomas Highland of Namibia, at $23^{\circ} 16^{\prime} \mathrm{S} 16^{\circ} 30^{\prime} \mathrm{E}, 1800 \mathrm{~m}$ above sea level. Each of the 13-m-diameter telescopes (Bernlöhr et al. 2003; Cornils et al. 2003) has a tessellated Davies-Cotton mirror of $107 \mathrm{~m}^{2}$ area and is equipped with a 960-photomultipliertube camera (Vincent et al. 2003) covering a large field of view of $5^{\circ}$ diameter. During stereoscopic observations, an arraylevel hardware trigger requires each shower to be detected by at least two telescopes simultaneously allowing for efficient suppression of the vast number of hadronic and muonic background events (Funk et al. 2004). The point source sensitivity reaches $1 \%$ of the flux of the Crab nebula for long exposures $(\approx 25 \mathrm{~h})$.

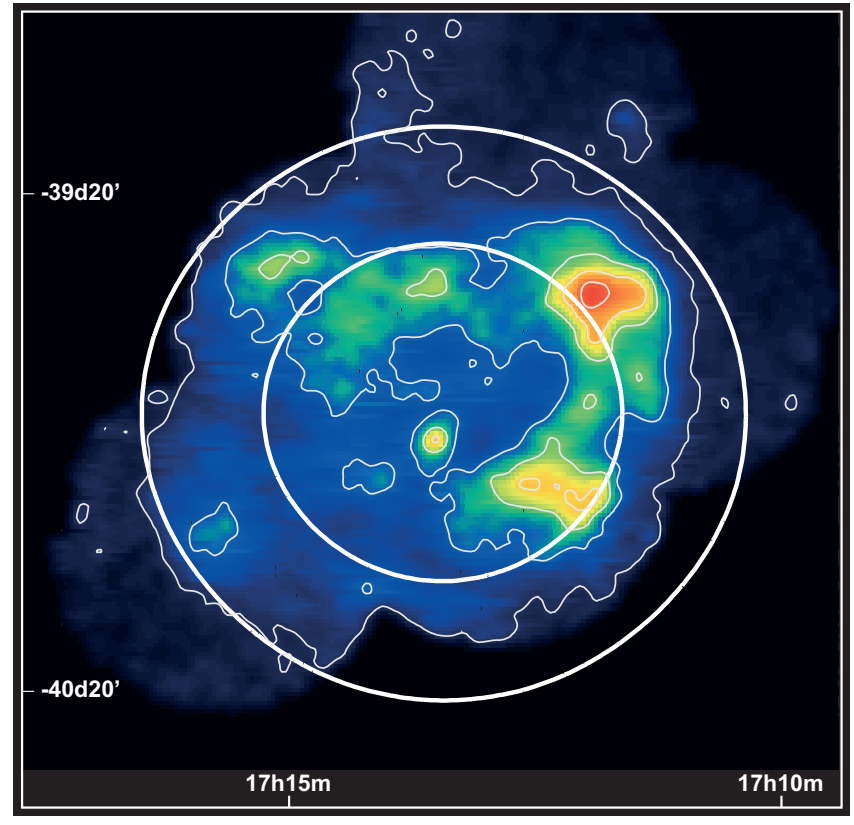

Fig. 1. X-ray image of RX J1713.7-3946 (colour scale and thin grey contour lines, 1-3 keV, from Uchiyama et al. 2002). The superimposed thick white contours indicate the $94 \%$ and $98 \%$ levels of the detectionefficiency weighted HESS exposure, given by the product of relative detection efficiency and the observation time. It can be seen that the relative gamma-ray detection efficiency between the centre region and the edges of the SNR differs only by $\approx 5 \%$.

The observations were mostly performed in wobble mode around the SNR centre. In this mode the telescopes were positioned such that the centre of the SNR was offset $\pm 0.7^{\circ}$ in declination or right ascension away from the pointing direction of the telescope system, changing to the next position every $28 \mathrm{~min}$. Towards the end of the observation campaign, pure onsource pointings in which the centre of the SNR was coincident with the system centre were additionally performed. In each of the five pointings the SNR RX J1713.7-3946, roughly $1^{\circ}$ in diameter, was fully contained in the $\approx 5^{\circ}$ field of view of the system. The resulting effective exposure distribution (the product of the detection efficiency and the exposure) is illustrated in Fig. 1, where the ASCA X-ray measurement (Uchiyama et al. 2002) is shown (colour scale and thin contour lines) with superimposed white, almost circular contours indicating the $94 \%$ and $98 \%$ levels of the effective exposure. The observation strategy for this data set combined with the detector efficiency results in a very flat plateau in the region of the SNR; from the centre to the boundaries the relative gamma-ray detection efficiency decreases by only about $5 \%$, which is a great advantage compared to ASCA, for example. Not only is the SNR fully contained in all of the five pointing positions, but one can also disregard for most purposes the modest change in relative detection efficiency from one region of the SNR to another.

\subsection{Data sample}

The data comprise a total exposure time of $40 \mathrm{~h}$. Rejecting data taken under bad weather conditions, $36 \mathrm{~h}$ of observation 

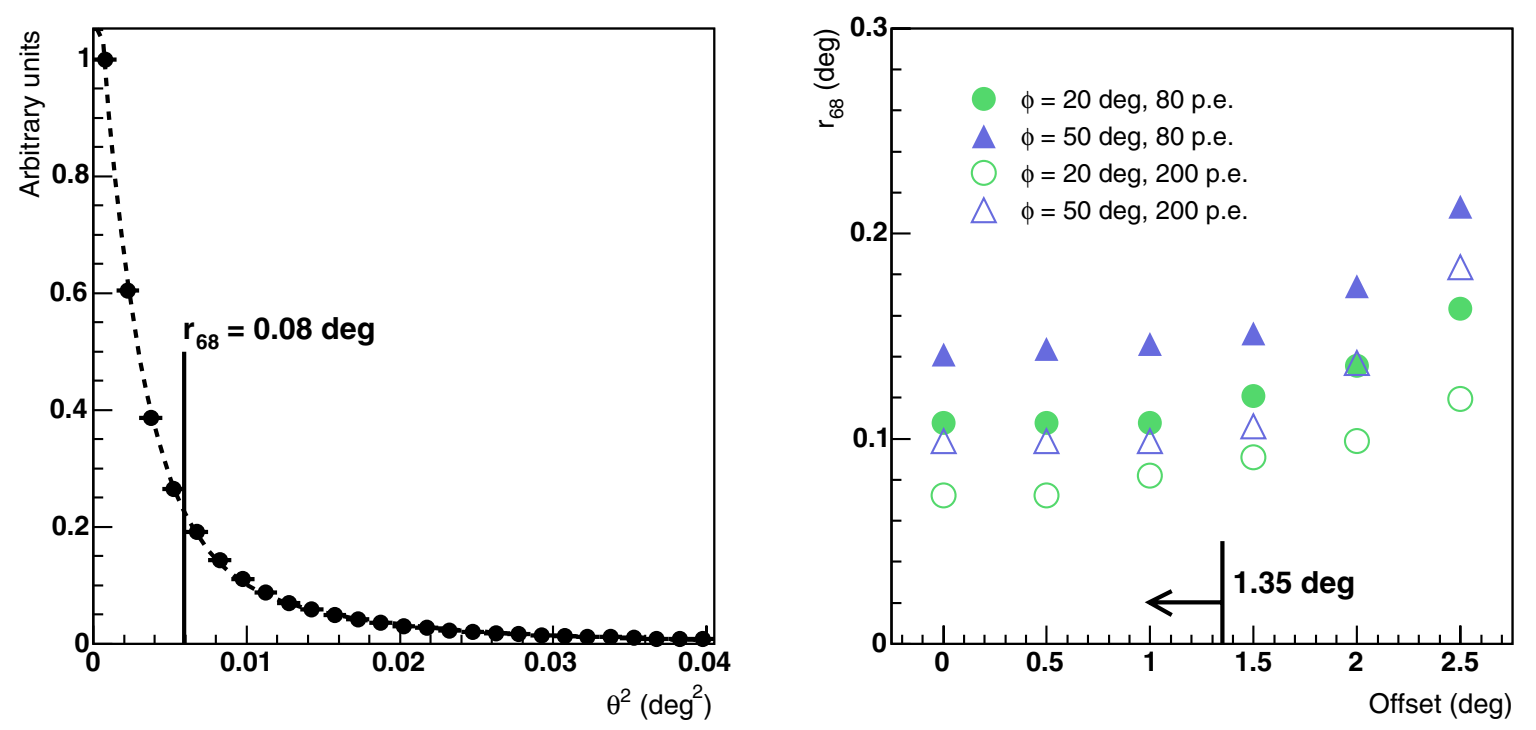

Fig. 2. Left hand side: squared angular distance between reconstructed and true direction for a Monte Carlo gamma-ray point source (pointspread function). A cut on the minimum size of images of 200 photo-electrons was applied. The simulated gamma rays followed a power law in energy with a photon index of 2 . The zenith angle distribution and offset of the source with respect to the telescope optical axis was matched to the actual data set of RX J1713.7-3946. The solid circles are the Monte Carlo histogram, the fit of a double Gaussian (dashed line) describes the point-spread function reasonably well. Indicated is the $68 \%$ containment radius of $0.08^{\circ}$ (obtained from the fit), which is taken as the resolution of the data set. Right hand side: $68 \%$ containment radii of the point-spread function determined from simulations as function of the offset between the source direction and telescope pointing. Shown are simulations for $50^{\circ}$ (triangles) and $20^{\circ}$ zenith angle (circles) for two different size cuts ( 80 and 200 photo-electrons). The maximum offset of the actual data set of $1.35^{\circ}$, given by the wobble displacement of $0.7^{\circ}$ and the approximate radius of the SNR of $0.65^{\circ}$, is indicated by the arrow.

time corresponding to $33 \mathrm{~h}$ of live time remain for the analysis. The zenith angle of observations ranged from $16^{\circ}$ to $56^{\circ}$ with a mean of $26^{\circ}$; it should be noted that about $68 \%$ of the data were taken at small zenith angles between $16^{\circ}$ to $26^{\circ}$. The energy threshold (defined by the peak gamma-ray detection rate for a given source spectrum after all gamma-ray selection cuts) of the system increases with zenith angle. For the observations presented here, assuming a spectrum appropriate for RX J1713.7-3946, the threshold was $\approx 180 \mathrm{GeV}$ at $16^{\circ}$, $\approx 340 \mathrm{GeV}$ at $40^{\circ}$, and $\approx 840 \mathrm{GeV}$ at $56^{\circ}$.

\subsection{Data preprocessing}

The analysis technique applied here is described in detail in Aharonian et al. (2005b). After calibration of the data (Aharonian et al. 2004a), tail-cuts image cleaning is applied and the shower images in each telescope are parametrised in terms of their centre of gravity and second moments (Hillas 1985). Stereoscopic event reconstruction based on the intersection of image axes yields the shower direction, providing a resolution of $\approx 0.1^{\circ}$ for individual gamma rays. Given the geometry of the shower, cuts (optimised on Monte Carlo gamma-ray simulations and $O F F$ source data, i.e. data without gamma-ray signal) are applied to select gamma-ray candidates and to suppress the vast hadronic background. The gammaray energy is estimated from the image intensity and the reconstructed shower geometry yielding a resolution of $\approx 15 \%$. There is one main difference between a point-source and an extended-source analysis. In the latter case, the cut on the squared distance of events to the assumed source location is greatly increased in order to reflect the large source extension. In that case the energy resolution worsens slightly (to $\$ 20 \%$ for a source of the size of RX J1713.7-3946) since an increased number of badly reconstructed events are included in the analysis.

\subsection{HESS performance for extended sources}

The large field of view of the HESS telescope system of $\approx 5^{\circ}$ diameter provides reasonable sensitivity for point sources at an angular distance up to $2^{\circ}$ from the pointing direction of the telescope system (the point-source off-axis sensitivity derived from Monte Carlo simulations has been confirmed via observations of the Crab nebula, see Aharonian et al. 2005c). Given the source diameter of up to $1.3^{\circ}$ and the offsets of $0.7^{\circ}$ between the centre of the SNR and the telescope pointing direction during observations, it is important that the gamma-ray point-spread function is well behaved and does not broaden significantly with increasing offset from the pointing direction. Figure 2 (left) shows the squared angular difference $\theta^{2}$ between the reconstructed and the true direction of a simulated point source. The initial simulations have been generated at a number of fixed zenith angles between $0^{\circ}$ and $70^{\circ}$. Taking then the zenith angle distribution of the actual data set and forming from that the weighted sum of the simulated $\theta^{2}$ distributions one obtains a resolution (taken as the $68 \%$ containment radius) of $0.08^{\circ}$. This is an order of magnitude smaller than the source diameter, which implies that in terms of angular resolution HESS is well suited for morphological studies of RX J1713.7-3946. The right hand side of Fig. 2 illustrates the dependence of the 
angular resolution on the offset between source position and pointing direction for two representative zenith angles of $20^{\circ}$ and $50^{\circ}$, for two different cuts on the minimum camera image size of 80 and 200 photo-electrons. It can be seen that the application of a higher cut on the image size improves the direction reconstruction by about $20 \%$ since only well defined camera images are used in the shower reconstruction, reducing fluctuation effects. However, the improved resolution is achieved at the expense of an increased energy threshold. In any case, for offsets smaller than $1.35^{\circ}$, which is the maximum offset under which parts of the SNR were observed, the resolution changes only slowly with offset and worsens by less than $10 \%$. It was furthermore demonstrated with observations of the point-like source PKS 2155-304 (Aharonian et al. 2005b), that simulated point-spread functions agree well with data distributions and are thus well understood.

The effective gamma-ray detection area depends on trigger conditions and analysis cuts. Well above the trigger threshold of the system, it is of the order of the area of the Cherenkov light pool (emitted by the secondary particle shower) on the ground. Typical effective area curves as a function of the offset angle between the gamma-ray source and the pointing direction of the system are shown in Fig. 3 for a zenith angle of $20^{\circ}$. The effective areas were determined from Monte Carlo simulations of a gamma-ray point source, accounting for the large extension of RX J1713.7-3946 by increasing the cut on the maximum distance to the assumed source position. Since for an extended source like RX J1713.7-3946 the flux has to be integrated over a larger solid angle than for point sources, the sensitivity of the system is reduced due to an increased background level. For moderate zenith angles and an integration region of $0.65^{\circ}$ radius around a gamma-ray source at $1^{\circ}$ offset from the pointing direction (matched to the data set described here), simulations reveal that, for a source like RX J1713.7-3946, the sensitivity drops roughly by a factor of four as compared to a point-source analysis.

\subsection{Analysis details}

The following section explains briefly the analysis applied to extract gamma-ray images and spectra of RX J1713.7-3946 from the data.

\subsubsection{Morphology}

The gamma-ray images shown throughout the paper represent, unless otherwise stated, gamma-ray excess counts with background subtracted. For the generation of these images a cut of 200 photo-electrons on the minimum camera image size in each telescope is applied to select a subset of events with superior angular resolution (see also Fig. 2 and Sect. 2.4). The background was estimated using about $160 \mathrm{~h}$ of HESS observations without any or only very faint gamma-ray sources in the field of view. All the events in these reference observations passing gamma-ray cuts are assumed to be gamma-ray like background events and are used to estimate the background for the given data set. For that purpose the set of OFF runs has been

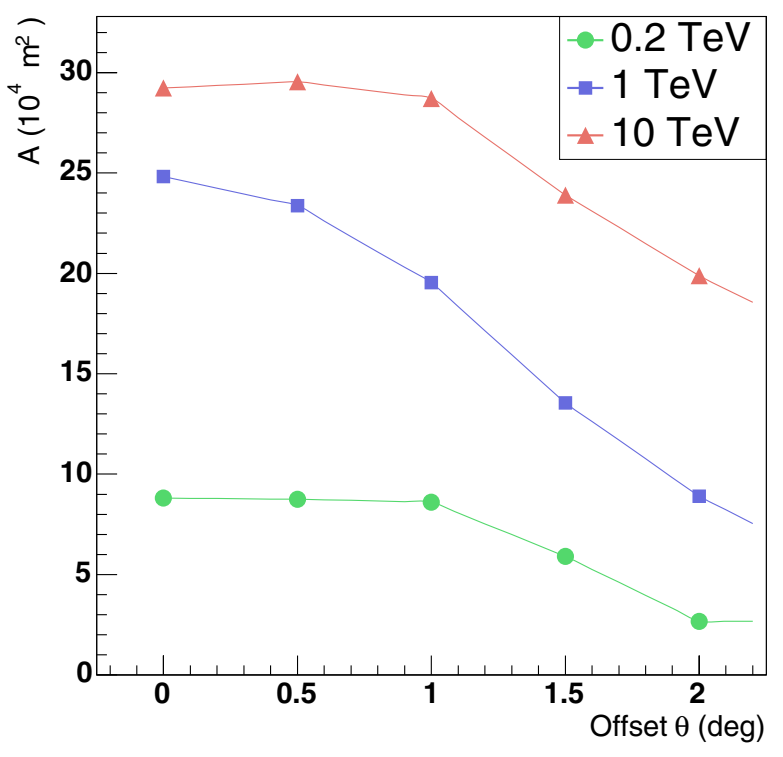

Fig. 3. Effective detection area $A$ for a moderate zenith angle of $20^{\circ}$ as a function of offset angle $\theta$ between the source location and the pointing direction of the system. Plotted are values at three representative energy values of $0.2 \mathrm{TeV}$ (near the post-cut trigger threshold), $1 \mathrm{TeV}$, and $10 \mathrm{TeV}$. The markers at $0^{\circ}, 0.5^{\circ}, 1^{\circ}, 1.5^{\circ}$, and $2^{\circ}$ represent the offset values for which simulations are available, in between two simulated offsets effective area values are interpolated linearly.

divided into distinct zenith angle bands to account for the dependance of the system's gamma-ray acceptance on observation altitude. Besides that, the acceptance depends only on the angular distance between shower and pointing direction and is to a very good approximation radially symmetric with respect to the pointing direction. Therefore, a radial 1D lookup (number of background events as a function of squared distance to the pointing centre) can be used in each zenith angle band for the background estimation. Given an observation at a certain zenith angle, a model background is created by selecting the 1D radial lookup from the zenith-angle band that matches the zenith angle of the observation. A 2D background map of the sky is then created by rotating the corresponding 1D lookup. Finally, the overall background map is created as the exposure weighted sum of the individual maps. A global normalisation factor $\alpha$ is calculated as ratio of the number of events in the data to the number of events in the background model, excluding regions that emit gamma rays. Figure 4 illustrates the validity of the approach for two HESS data sets, RX J1713.7-3946 (upper panel) and PKS 2155-304 (lower panel), the latter being a point source for HESS. Shown are slices along right ascension through the source centres. Overlaid on both data curves are the normalised background models of the whole data sets. In both cases, at different regions in the sky, for an extended and a point-like gamma-ray source, there is clearly a good match between model and data in regions outside the gamma-ray sources.

Images of the gamma-ray excess are obtained by subtracting the normalised background model from the data in each bin $i$ of the 2D map:

$N_{\text {excess }, i}=N_{\text {data }, i}-\alpha N_{\text {background }, i}$. 


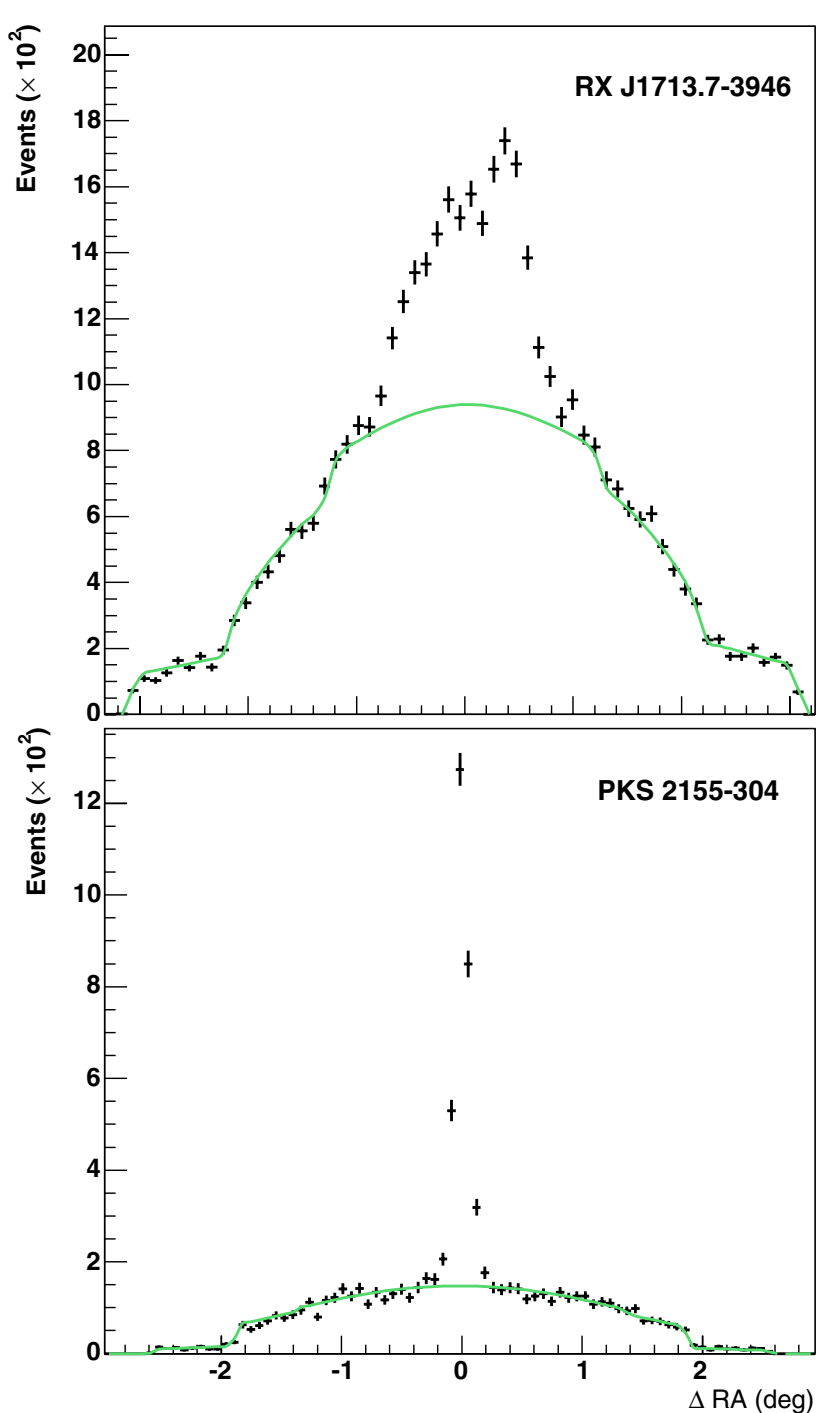

Fig. 4. As illustration of the good match between data and background model, the data set of RX J1713.7-3946 (upper panel) is compared to the high statistics data set of PKS 2155-304 (lower panel) which appears as a point source for HESS. Shown are $1.3^{\circ}$ and $0.2^{\circ}$ slices along the RA axis through the centre of RX J1713.7-3946 and PKS 2155-304, respectively, for the data (black) and the background model (green). The good match both in shape and absolute level between the two curves illustrates the validity of the approach. The steps in the distributions (in the case of RX J1713.7-3946 at $\pm 1.1^{\circ}$ and $\pm 2.1^{\circ}$ ) are artefacts of the analysis: the usable range in the field of view of every observation was restricted to a radius of $2^{\circ}$ (out of $2.5^{\circ}$ physical camera radius) around the camera centre to allow only for well reconstructed events with good camera acceptance, and the figure combines data from different pointings.

Subsequently, these images are smoothed with a Gaussian to reduce statistical fluctuations.

Typically the standard deviation for the smoothing is matched to the resolution of the data set, namely $30 \%-50 \%$ of the $68 \%$ containment radius of the point-spread function. Here, for the data of RX J1713.7-3946, a smoothing radius of $2^{\prime}$ is used. The resulting count maps are in units of integrated excess counts per Gaussian sigma of the smoothing function.
No correction for the falloff of detection efficiency towards the edges of the field of view is applied because in the region of interest, around the SNR RX J1713.7-3946, the variation in gamma-ray acceptance is negligible (see Fig. 1).

\subsubsection{Spectral analysis}

For the spectral analysis, the HESS standard cut on the image size of 80 photo-electrons is applied to the data. To obtain a spectrum of a certain region in the sky, all events with reconstructed direction in that particular region are considered as ON events. A complication arises for the background estimation. The gamma-ray acceptance and therefore the background level depend strongly on energy; one cannot, as for the image generation, simply use a 1D radial lookup (which is integrated over all energies) as a background estimate. Instead, the acceptance lookups would have to be generated in energy bins which in practice is difficult to handle. Another approach was applied here for the spectral analysis: background (OFF) events were selected from the same field of view, from the same data run, by selecting regions of the same size and form as the ON region, but displaced on a circle around the pointing direction of the system. The circle is chosen such that the OFF regions are at exactly the same offset (that is, at the same angular distance to the pointing direction) as the $\mathrm{ON}$ region. A minimum distance between the $\mathrm{ON}$ and $\mathrm{OFF}$ regions of $0.1^{\circ}$ is required to avoid gamma-ray contamination. Furthermore, known gamma-ray sources in the field of view not associated with the test region are excluded from the OFF regions. This approach ensures that background events are taken at the same zenith and offset angles, which is crucial because of the dependence of the effective detection areas upon these two quantities, and it uses more or less the same region of sky, with similar night-sky-background noise. For an object of the size of RX J1713.7-3946 this results in one ON and OFF region, the latter being simply the reflection of the former at the system centre. This is illustrated in Fig. 5, where the OFF regions used for each observation position are drawn.

After the geometrical selection, in order to obtain a differential gamma-ray flux $\mathrm{d} N / \mathrm{d} E$ in units of $\left(\mathrm{TeV}^{-1} \mathrm{~cm}^{-2} \mathrm{~s}^{-1}\right), \mathrm{ON}$ and OFF events ( $N_{\mathrm{ON}}$ and $\left.N_{\mathrm{OFF}}\right)$ are binned logarithmically in energy and divided by the mean effective area and the exposure time in each bin. The energy dependent effective area is determined for each data run, for the corresponding zenith $\left(\theta_{\mathrm{z}}\right)$ and offset $(\theta)$ angle range, multiplied by the live time of the run and added up. Then, for each energy bin $i$, the bin entries are divided by the width $\left(\Delta_{i}\right)$ of that bin to obtain a differential flux value. The differential flux results from subtracting the differential OFF- from the ON-flux histogram:

$\left(\frac{\mathrm{d} N}{\mathrm{~d} E}\right)_{i}=\frac{N_{\mathrm{on}, i}}{\Delta_{i} \Sigma_{\mathrm{runs}} T A_{i}\left(\theta_{\mathrm{z}}, \theta\right)}-\alpha_{i} \frac{N_{\mathrm{OFF}, i}}{\Delta_{i} \Sigma_{\mathrm{runs}} T A_{i}\left(\theta_{\mathrm{z}}, \theta\right)}$.

The normalisation factor $\alpha_{i}$ is determined from the ratio of the areas of the ON and OFF regions used during analysis of the whole data set and takes the exposure of the different observations into account.

An alternative approach is the event-by-event usage of the effective area, which served as a systematic check. Rather than 


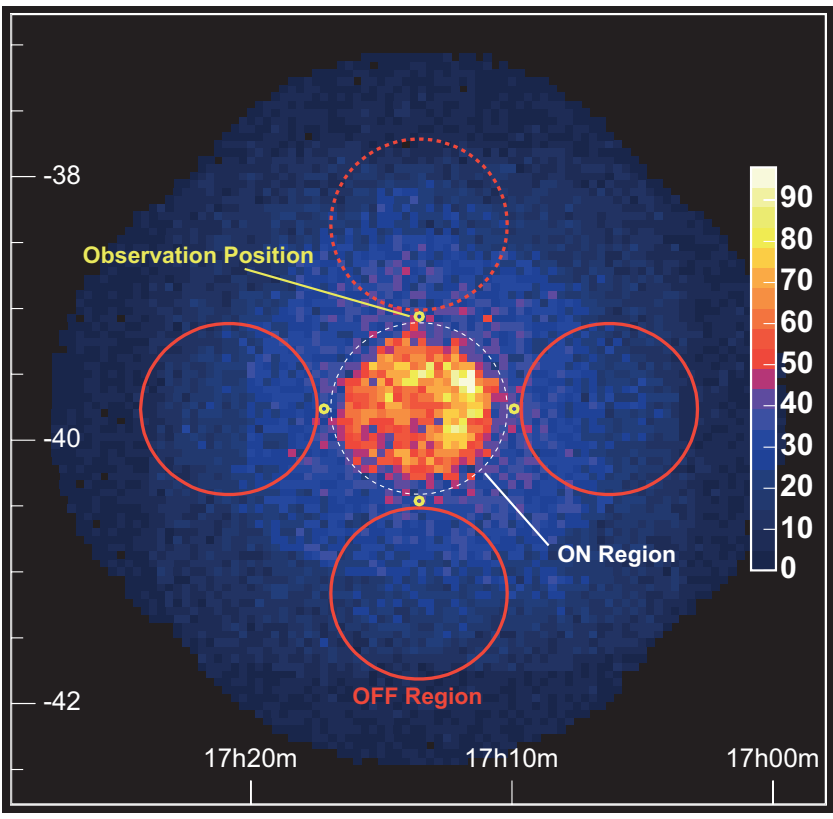

Fig. 5. Count map of gamma-ray candidates for the region around RX J1713.7-3946. A size cut of 200 photo-electrons on the camera images was applied. The bins are uncorrelated and the background is not subtracted. The white dashed circle indicates the region used to extract the spectrum of the whole SNR (the ON region), the red circles indicate the OFF regions, the regions used for background estimation for the spectrum in each of the four wobble observation positions, which are marked as yellow circles. The dashed red circle to the north of the SNR indicates an OFF region that was not used in the spectral analysis because it contains a gamma-ray source discovered in the HESS Galactic plane survey (Aharonian et al. 2006).

determining a mean effective area for the whole data set, each event is weighted with the inverse of the effective area, taking the event zenith angle, offset and energy.

A complication arises from the dependence of the effective detection area on zenith angle and offset. The Monte Carlo effective areas are generated at certain discrete zenith angles and offsets. When generating the mean effective area corresponding to a certain zenith angle and offset range, the effective area is interpolated linearly between the simulation values (see Fig. 3, where the markers indicate values available from simulations and the lines are the linear interpolations).

\section{Results}

With the HESS data set, the morphology of RX J1713.7-3946 and its spectrum are resolved with high precision. Given that this is the first in depth analysis of such an extended source in VHE gamma rays, we present in the following first selected examples of extensive systematic tests that were performed in order to assure the stability of the analysis and then discuss the results.

\subsection{Morphology}

When analysing the morphology of an extended source, one aims for the best possible resolution with, at the same

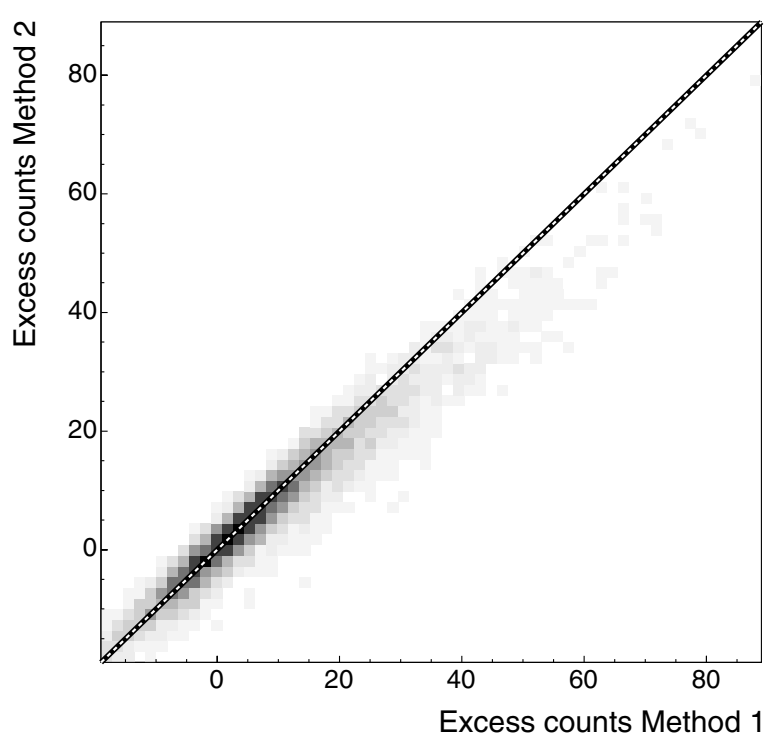

Fig. 6. Correlation between the standard calibration, event reconstruction, and background reduction method (Method 1) and an alternative, completely independent approach (Method 2, see main text for more details). The black solid and white dashed lines run through the origin with a slope of 1 . A linear correlation is clearly visible, deviations from the line originate from different cut efficiencies of the two methods.

time, sufficient event statistics. In order to explore image structures and their stability, the data set was analysed using the same calibration and analysis software, but applying different sets of cuts (like accepting only three- and four-telescope events and events with image amplitudes larger than 80, 200, and 400 photo-electrons), which resulted in different resolutions and different event statistics. Another important issue is the appropriate modelling and subtraction of the background. As a systematic test, alternative background models have been applied and the results compared. First, a set of OFF runs, taken at the same zenith angles with very similar night-sky noise as the ON runs, has been used. Furthermore, the morphology was cross-checked using a completely independent calibration and analysis approach, not only for the reconstruction of the shower geometry but also for background estimation. Rather than using standard (Hillas) parameters for image parametrisation and reconstructing shower geometry based on these parameters, this approach is based on a 3D modelling of Cherenkov photon emission during the shower development in the atmosphere assuming rotational symmetry, thereby predicting pixel amplitudes (for more details, see Lemoine-Goumard \& de Naurois 2005). A comparison of the two analysis methods is shown in Fig. 6; plotted is the correlation of gamma-ray excess counts for the sky region around RX J1713.7-3946. A linear correlation is clearly apparent illustrating the good agreement of the two independent methods.

With the systematic tests mentioned above it could be shown that the main features of the gamma-ray morphology are stable when analysed with different cuts, different background models as well as with independently determined calibration coefficients and alternative analysis methods. 


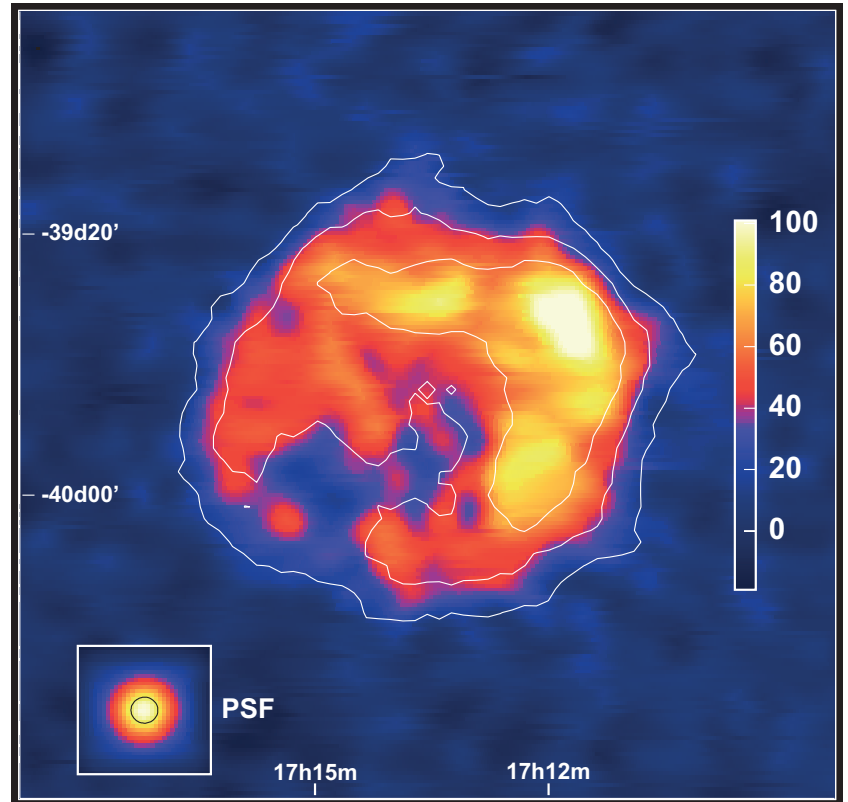

Fig. 7. Gamma-ray image of RX J1713.7-3946. The linear colour scale is in units of excess counts (see Sect. 2.5.1 for a description of image generation). The white contour lines indicate the significance of the different features, the levels are linearly spaced and correspond to 5,10 , and $15 \sigma$, respectively. The significance of each point has been calculated assuming a point source at that position, integrating events within a circle of $0.1^{\circ}$ radius. In the lower left hand corner a simulated point source is shown as it would appear in this particular data set (taking the point-spread function and the smoothing into account) along with a black circle of $2^{\prime}$ radius denoting the $\sigma$ of the Gaussian the image is smoothed with.

Figure 7 shows a $2^{\circ} \times 2^{\circ}$ field of view around RX J1713.73946. A cut on the image size at 200 photo-electrons was applied resulting in a superior resolution of $0.08^{\circ}$ (see Fig. 2). The corresponding energy range is $\approx 300 \mathrm{GeV}$ to $\approx 40 \mathrm{TeV}$. This image of RX J1713.7-3946 confirms with much higher statistics the 2003 HESS measurement, shown for example in Fig. 1 of Aharonian et al. (2004b). There is no evidence for time variability, as expected for an object of the size of RX J1713.7-3946. The overall gamma-ray appearance resembles a shell morphology with bright emission regions in the western and northwestern part where the SNR is believed to impact molecular clouds (Fukui et al. 2003; Moriguchi et al. 2005). It is worth noting that there is a possible gamma-ray void in the central-southeastern region. The cumulative significance for the whole SNR is about $39 \sigma$ with these hard cuts, which corresponds to an excess of $\approx 7700$ events from the region of RX J1713.7-3946. Drawn as white lines in Fig. 7 are in addition the contours of significance of the gamma-ray signal (levels correspond to 5, 10, and $15 \sigma$ ). The significance has been calculated considering events that fall within an angle of $0.1^{\circ}$ of each trial source position. Thus, the contours quantify the significance for each point as if there was a point source at that position. The background estimate was derived from OFF runs as described in Sect. 2.5.1. The brightest parts of the SNR exceed $20 \sigma$. Except for the void structure, where the significance
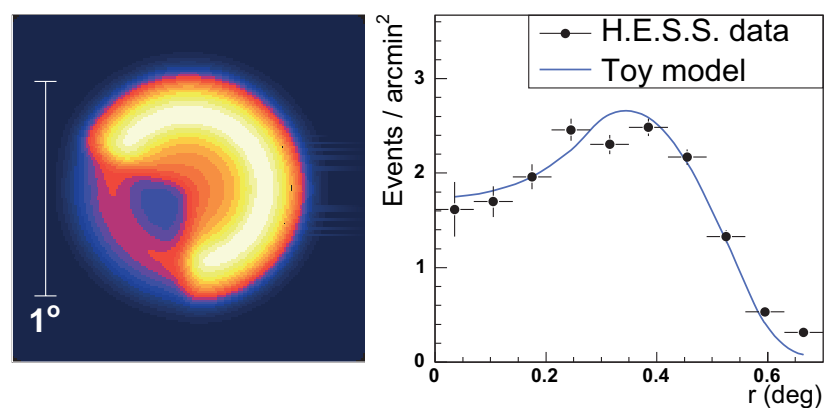

Fig. 8. Shown is a simple geometrical model for the emission from a thick sphere matched to the dimensions and relative fluxes of RX J1713.7-3946. Left: 2D projection of a thick and spherical radiating shell, $1^{\circ}$ in diameter, smoothed with the HESS point-spread function. Adapted empirically to match the radial shape of the HESS data set, the dimensions of the geometrical sphere are $5.5 \mathrm{pc}$ for the inner and $10 \mathrm{pc}$ for the outer boundary if one assumes a distance of $1 \mathrm{kpc}$ to the source, the presumed distance to RX J1713.7-3946. The emissivity in the northern, western, and southwestern part is a factor of two higher than in the southeast and east. Right: radial profile from the geometrical model compared to the HESS data profile of RX J1713.7-3946. The centre coordinates used for the data plot are $\alpha_{\mathrm{J} 2000}=17 \mathrm{~h} 13 \mathrm{~m} 33.6 \mathrm{~s}, \delta_{\mathrm{J} 2000}=-39^{\circ} 45^{\prime} 36^{\prime \prime}$. The geometrical model profile has been scaled to the same area as the data profile.

just exceeds $5 \sigma$, most of the remaining emission regions are well above $10 \sigma$.

From the gamma-ray image presented here it is clear that the emission regions cannot be distributed homogeneously in the sphere RX J1713.7-3946. The image is neither rotational symmetric nor does it exhibit a shallow peak towards the centre. Instead, a shell seems to be apparent in the northern, and western to southwestern part. Apart from that, there is more or less uniform emission found in the rest of the SNR with a slight flux increase towards the southeastern boundary. This resembles very much the image one would expect from a thick spherical shell radiating gamma rays with enhanced emission on one side, as is illustrated in Fig. 8 where a geometrical model of a thick radiating sphere is presented. The good match in shape between the data and the toy-model profile lends support to the assumption that indeed it is the shell of RX J1713.7-3946 which radiates gamma rays.

Figure 9 shows three images of RX J1713.7-3946 in three distinct energy bands, $E<0.6 \mathrm{TeV}, 0.6 \mathrm{TeV}<E<$ $1.4 \mathrm{TeV}$, and $1.4 \mathrm{TeV}<E$ (left to right). The energy ranges were chosen such that each band represents a third of the data set. Note that the angular resolution of all three images is roughly the same which makes them readily comparable. The signal-to-noise ratio of the low-energy image is evidently smaller. The shell-like morphology of the SNR is slightly blurred by fluctuations. Correspondingly, the significance contours indicate that only the bright northwestern half is significant in this energy band. In contrast, the whole remnant sticks out significantly in the two higher-energy bands. Most of the northwestern parts exceed $10 \sigma$, the brightest spots even exceed $15 \sigma$ for energies beyond $1.4 \mathrm{TeV}$.

From the visual impression the remnant does seem to emit gamma rays more uniformly with increasing energy. However, 

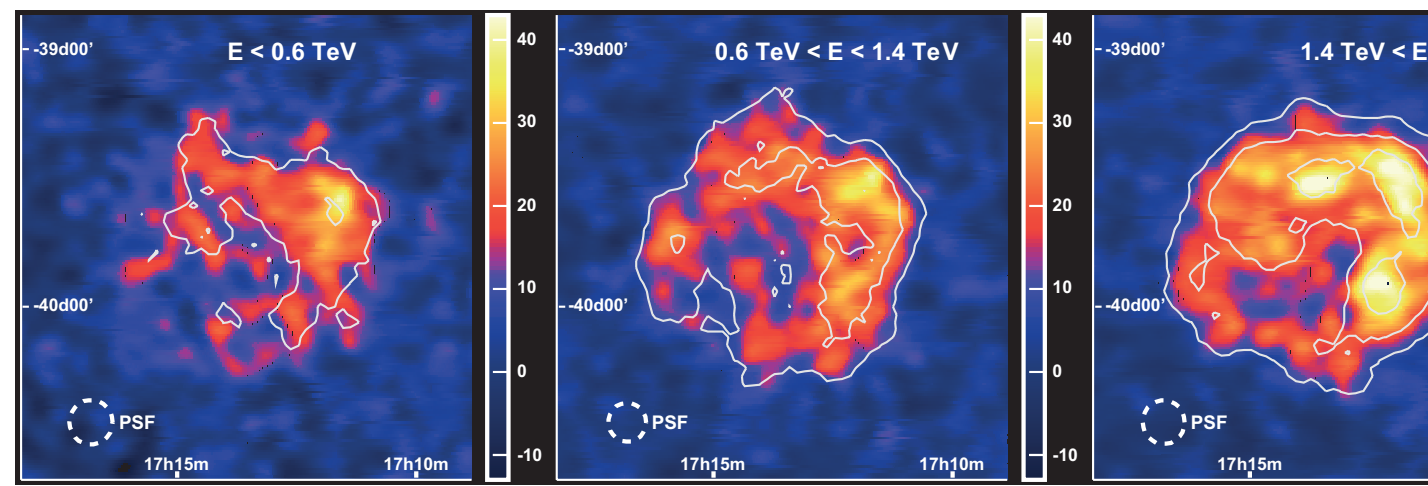

Fig. 9. Morphology of RX J1713.7-3946 as it appears at different energies. Shown from left to right are gamma-ray excess images with energies of $E<0.6 \mathrm{TeV}, 0.6 \mathrm{TeV}<E<1.4 \mathrm{TeV}$, and $1.4 \mathrm{TeV}<E$. Drawn additionally as white lines are contours of significance, linearly spaced at 5 , $10,15 \sigma$ (as in Fig. 7). Note the increase in the signal-to-noise ratio with increasing energy. The energy bands were chosen such that each band represents about a third of the full data set (taking events after cuts). Furthermore, all three images were smoothed with a Gaussian of $2^{\prime}$, which makes them directly comparable to each other, and to Fig. 7. The resolution in each energy band is indicated in the lower left hand corner of the images; the three data subsets have comparable resolutions of $\approx 0.08^{\circ}$ (the resolution of the intermediate energy band is about $6 \%$ better). This might be counter-intuitive, given that at larger energies camera images get bigger and fluctuation effects become negligible thereby improving the energy and direction resolution. However, in this case that effect is compensated by the increasing mean zenith angle of the large-energy events.

within errors, the radial shape appears to be the same in all three energy bands, as seen from the radial excess profiles around the centre of the SNR shown in Fig. 10. The morphology does not change significantly with energy. This is qualitatively compatible with the results of the spatially resolved spectral analysis (see Sect. 3.2).

\subsection{Energy spectra}

The energy spectrum of RX J1713.7-3946 was measured with HESS over a large energy range. Systematic tests on the shape and characteristics of the energy spectrum of the whole SNR included application of a slightly different spectral analysis technique, different background models, analysis in distinct data subsets like small and large zenith angles and the five observation positions, analysis applying different cuts on image intensity and telescope multiplicity, investigation of the influence of the exact binning, of the energy estimation and the fit to the effective area histograms obtained from simulations. Furthermore, the results were cross-checked with the independent calibration and analysis scheme mentioned in Sect. 3.1. Representative examples for these systematic tests are shown in Fig. 11, where the spectrum of the whole SNR is plotted using the standard background estimation from the same field of view, compared to a completely independent background estimation based on OFF runs, and to the independent analysis chain (Lemoine-Goumard \& de Naurois 2005). The three spectra are found to be well compatible with each other.

In order to obtain a quantitative estimate for the systematic error on each flux point, the background estimation, the spectral analysis technique (event-wise effective area weighting or average effective area determination per data run) and the absolute energy scale of the experiment were considered as dominant contributions. For the background modelling, an uncertainty of $\Delta \alpha=1 \%, \alpha$ being the background normalisation factor, was derived from the RX J1713.7-3946 data

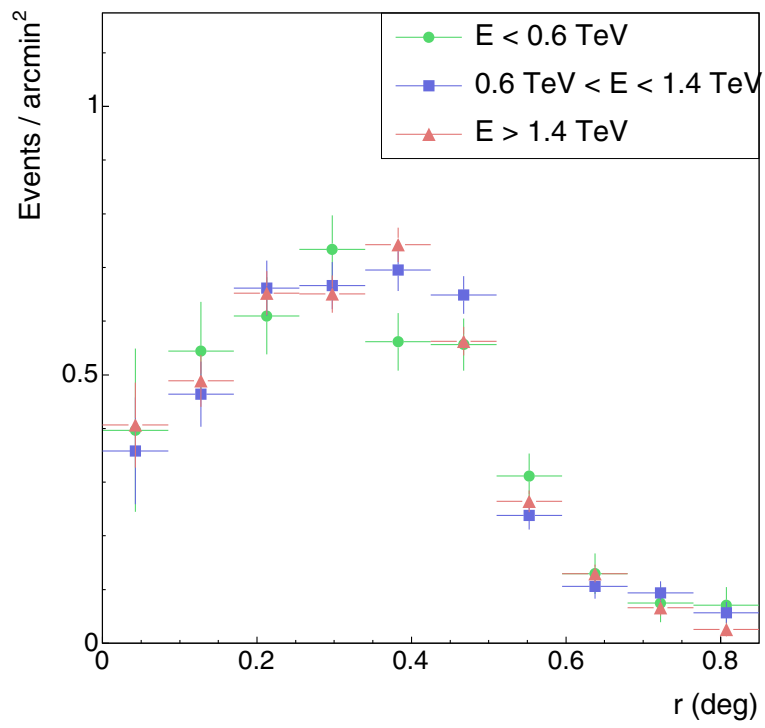

Fig. 10. Radial profiles around the centre of the $\operatorname{SNR}\left(\alpha_{\mathrm{J} 2000}=\right.$ $\left.17 \mathrm{~h} 13 \mathrm{~m} 33.6 \mathrm{~s}, \delta_{\mathrm{J} 2000}=-39^{\circ} 45^{\prime} 36^{\prime \prime}\right)$ in the three energy bands plotted in Fig. 9, generated from the raw (that is, not smoothed) gamma-ray excess images. The intermediate and high-energy band images have improved signal-to-noise ratios. The radial profiles from these energy bands have been scaled such that they have the same area as the lowenergy profile (scaling factors are $\approx 0.87$ and $\approx 0.65$, respectively), to enable direct comparisons.

set by comparing the standard analysis to the analysis using an independent background model derived from OFF runs, as mentioned above. It should be noted that the $1 \%$ variation found in the total number of background counts is compatible with being due to a statistical variation and thus must be regarded as an upper limit. Taking the systematic uncertainty due to the background level and the two spectral analysis techniques, an energy dependent systematic error was obtained by analysing the data set six times - scaling $\alpha$ by 


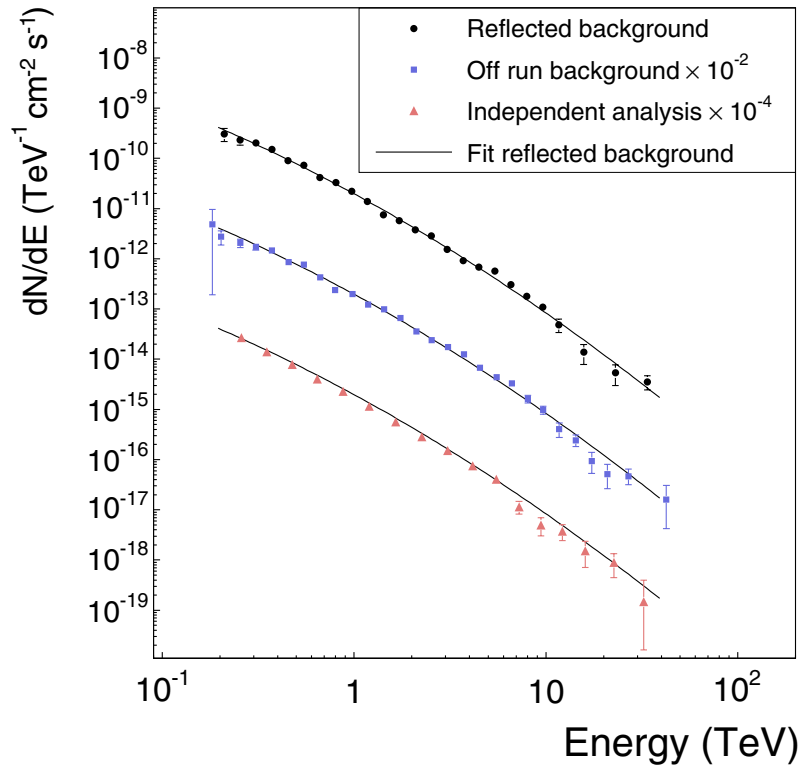

Fig. 11. Shown are three spectra that were produced to explore the systematic uncertainties. The alternative spectra (blue squares and red triangles) were scaled by factors of $10^{-2}$ and $10^{-4}$, respectively, for presentation reasons. Upper set: standard analysis and standard background modelling using a background position from the same field of view, with the exact same shape and distance to the pointing direction as the signal region, but opposite to it, on the other side of the pointing direction. Middle set: alternative spectral analysis technique (event-wise effective area weighting, see Sect. 2.5.2) with an independent background estimate taken from OFF runs. The background regions in the OFF data were again selected such that they have the same shape and distance to the pointing direction as the signal region in the RX J1713.7-3946 observations. Lower set: spectrum produced applying an independent analysis chain. The background was determined similarly as for the upper set. A third spectral analysis technique was applied here, described in Piron et al. (2001). Plotted as black line on top of all three spectra to guide the eye is the best fit of a power law with energy dependent photon index to the spectrum shown in the upper set (see Table 1 for details). The error bars on the spectral points denote $\pm 1 \sigma$ statistical errors.

$(1+[-1,0,+1] \times \Delta \alpha)$ and applying both analysis techniques. The rms of the resulting six flux points in each energy bin was taken as the systematic uncertainty. The uncertainty in the energy scale is a global uncertainty which might cause a shift of the whole spectrum to lower or larger energies. It is due to uncertainties in the atmospheric transmission models used in simulations (see Funk et al. 2004) and uncertainties in the light collection efficiencies of the telescopes. The combined error is estimated to be $20 \%$.

When fitting a power law with index $\Gamma$ to the spectrum, the systematic error on the integral flux obtained from the fit function is conservatively estimated to be $25 \%$, on the fit index it is $\Delta \Gamma=0.1$.

The spectrum of the whole SNR was determined by integrating events within $0.65^{\circ}$ radius around the centre of the $\mathrm{SNR}, \alpha_{\mathrm{J} 2000}=17 \mathrm{~h} 13 \mathrm{~m} 33.6 \mathrm{~s}, \delta_{\mathrm{J} 2000}=-39^{\circ} 45^{\prime} 36^{\prime \prime}$. OFF events were selected from a reflected region in the same field of view (see Sect. 2.5.2 for explanation and Fig. 5 for illustration). To ensure optimum match in the offset distributions of $\mathrm{ON}$ and

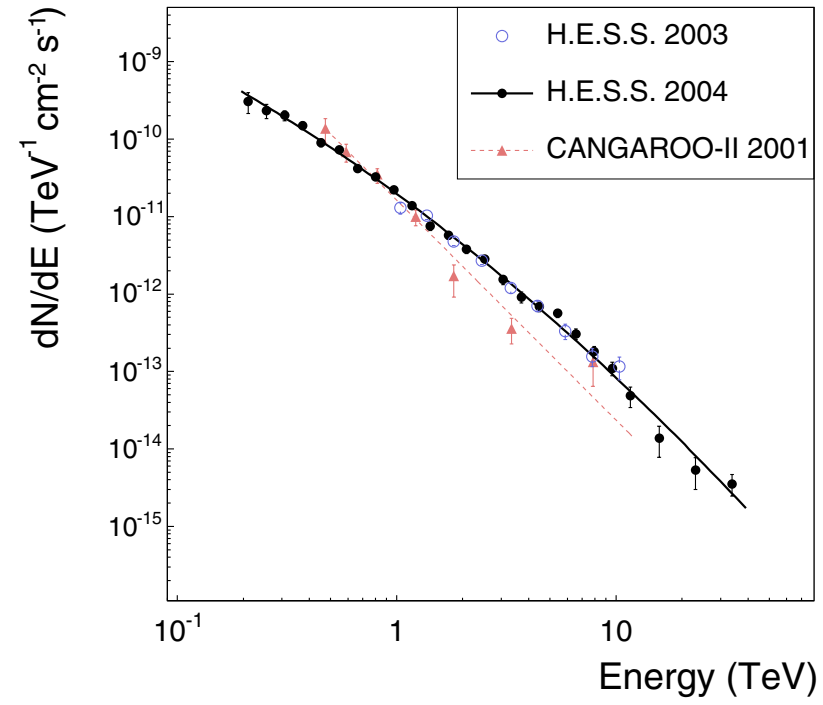

Fig. 12. Differential gamma-ray energy spectrum of RX J1713.73946, for the whole region of the SNR (solid black circles). The best fit of a power law with energy dependent photon index is plotted as black line. For comparison the HESS 2003 data points are also shown (blue open circles). Note the vast increase in energy coverage due to the increased sensitivity of the complete telescope array. The spectrum ranges now from $190 \mathrm{GeV}$ to $40 \mathrm{TeV}$, spanning more than two decades in energy. The data points reported by the CANGAROO-II collaboration (Enomoto et al. 2002) for the northwestern part of the remnant are also shown as red triangles, the corresponding best fit result as dashed red line. Error bars are $\pm 1 \sigma$ statistical errors.

OFF events, runs taken directly on the source, where no appropriate OFF region can be selected in the same field of view, were discarded from the spectral analysis. Accordingly, the total live time reduced slightly to $30.5 \mathrm{~h}$. A size cut of 80 photoelectrons was applied for the spectral analysis. This results in a cumulative significance of $31 \sigma$ corresponding to $\approx 15400$ excess events (normalisation factor $\alpha=1.3$ ).

The resulting spectrum of the whole SNR is shown in Fig. 12. The data is in excellent agreement with the previous measurement in 2003, which covered the energy range from $1 \mathrm{TeV}$ to $10 \mathrm{TeV}$. The latest data span more than two orders of magnitude in energy, from $190 \mathrm{GeV}$ to $40 \mathrm{TeV}$. The best fit of a power law with energy dependent photon index is plotted (the exact formula is given below). It describes the data reasonably well. Table 1 summarises fits of different spectral shapes to the data. Three alternative shapes have been used: a power law with an exponential cutoff $E_{\mathrm{c}}$,

$\frac{\mathrm{d} N}{\mathrm{~d} E}=I_{0}\left(\frac{E}{1 \mathrm{TeV}}\right)^{-\Gamma} \exp \left(-\frac{E}{E_{\mathrm{c}}}\right)$

a power law with an energy dependent exponent,

$\frac{\mathrm{d} N}{\mathrm{~d} E}=I_{0}\left(\frac{E}{1 \mathrm{TeV}}\right)^{-\Gamma+\beta \log \frac{E}{1 \mathrm{TeV}}}$,

and a broken power law (transition from $\Gamma_{1}$ to $\Gamma_{2}$ at break energy $E_{\mathrm{B}}, S$ quantifies the sharpness of the transition),

$\frac{\mathrm{d} N}{\mathrm{~d} E}=I_{0}\left(\frac{E}{E_{\mathrm{B}}}\right)^{-\Gamma_{1}}\left(1+\left(\frac{E}{E_{\mathrm{B}}}\right)^{1 / S}\right)^{S\left(\Gamma_{2}-\Gamma_{1}\right)}$. 
Table 1. Fit results for different spectral models. The differential flux normalisation $I_{0}$ and the integral flux above $1 \mathrm{TeV}(I(>1 \mathrm{TeV}))$ are given in units of $10^{-12} \mathrm{~cm}^{-2} \mathrm{~s}^{-1} \mathrm{TeV}^{-1}$ and $10^{-12} \mathrm{~cm}^{-2} \mathrm{~s}^{-1}$, respectively. The power-law fit is clearly an inappropriate description of the data, a power law with an exponential cutoff (row 2), a power law with an energy dependent photon index (row 3), and a broken power law (row 4; in the formula, the parameter $S=0.4$ describes the sharpness of the transition from $\Gamma_{1}$ to $\Gamma_{2}$ and it is fixed in the fit) are equally likely descriptions of the HESS data. Note that when fitting a broken power law to the data, some of the fit parameters are highly correlated.

\begin{tabular}{|c|c|c|c|c|c|c|}
\hline Fit Formula & \multicolumn{4}{|c|}{ Fit Parameters } & $\overline{\chi^{2} \text { (d.o.f.) }}$ & $\overline{I I(>1 \mathrm{TeV})}$ \\
\hline$I_{0} E^{-\Gamma}$ & $I_{0}=17.1 \pm 0.5$ & $\Gamma=2.26 \pm 0.02$ & & & $85.6(23)$ & $13.5 \pm 0.4$ \\
\hline$I_{0} E^{-\Gamma} \exp \left(-E / E_{\mathrm{c}}\right)$ & $I_{0}=20.4 \pm 0.8$ & $\Gamma=1.98 \pm 0.05$ & $E_{\mathrm{c}}=12 \pm 2$ & & $27.4(22)$ & $15.5 \pm 1.1$ \\
\hline$I_{0} E^{-\Gamma+\beta \log E}$ & $I_{0}=19.7 \pm 0.6$ & $\Gamma=2.08 \pm 0.04$ & $\beta=-0.30 \pm 0.04$ & & $25.5(22)$ & $15.6 \pm 0.7$ \\
\hline$I_{0} E / E_{\mathrm{B}}^{-\Gamma_{1}}\left(1+E / E_{\mathrm{B}}^{1 / S}\right)^{S\left(\Gamma_{2}-\Gamma_{1}\right)}$ & $I_{0}=0.4_{-0.2}^{+0.6}$ & $\Gamma_{1}=2.06 \pm 0.05$ & $\Gamma_{2}=3.3 \pm 0.5$ & $E_{\mathrm{B}}=6.7 \pm 2.7$ & $26.2(21)$ & $15.4 \pm 0.8$ \\
\hline
\end{tabular}

In all cases, $I_{0}$ is the differential flux normalisation, the energies $E$ are normalised at $1 \mathrm{TeV}$ and photon indices are specified with $\Gamma$.

All three alternative shapes describe the data significantly better than the pure power law. However, among the alternative spectral shapes, none is significantly favoured over the others. At the highest energies, above $10 \mathrm{TeV}$, there is still a significant gamma-ray flux in excess of $6 \sigma$. It should be noted, though, that in order to draw strong conclusions about the high-energy shape of the spectrum, more data with better statistics at the high-energy end of the spectrum are needed.

The spectrum reported by the CANGAROO-II collaboration (Enomoto et al. 2002) for the northwest part of the SNR is also shown in Fig. 12. From a power-law fit to the data they quoted a photon index $\Gamma=2.84 \pm 0.15$ (statistical) \pm 0.20 (systematic) and a differential flux normalisation at $1 \mathrm{TeV}$ $I_{0}=(1.63 \pm 0.15 \pm 0.32) \times 10^{-11} \mathrm{~cm}^{-2} \mathrm{~s}^{-1} \mathrm{TeV}^{-1}$. The difference between the two spectra is somewhat larger than the quoted errors of the measurements. However, the CANGAROO-II spectrum is only for a part of the remnant. Moreover we note that the CANGAROO-II collaboration has recently revised their systematic errors upwards. For example, the Galactic Centre photon index, which was initially given as $4.6 \pm 0.5$ (Tsuchiya et al. 2004), was recently quoted as $4.6_{-1.2}^{+5.0}$ (Katagiri et al. 2005).

Figure 13 illustrates the three spectral shapes that were found to describe the data reasonably well. The three curves are extrapolated to $1 \mathrm{GeV}$ to compare them with the EGRET upper limit on the energy flux of $4.9 \times 10^{-11} \mathrm{erg} \mathrm{cm}^{-2} \mathrm{~s}^{-1}$, ranging from $1 \mathrm{GeV}$ to $10 \mathrm{GeV}$, centred at $2.9 \mathrm{GeV}$. The limit was determined at the HESS position of RX J1713.7-3946 by modelling and subtracting the known EGRET source 3EG 1714-3857 (Hartman et al. 1999), assuming that 3EG 1714-3857 is not linked to the gamma-ray emission of RX J1713.7-3946. Since the HESS location is in close vicinity of 3EG 1714-3857 (actually it is overlapping), this procedure could only be carried out successfully above $1 \mathrm{GeV}$. The systematic error band for the HESS data was obtained as described above. It is centred on the mean value of the three fit curves and represents the systematic error due to background uncertainties only. The energy scale is an energy independent uncertainty; its scale and the direction the curve is shifted to are marked with a blue arrow at one representative position (at $15 \mathrm{TeV}$ ). It is worth noting that

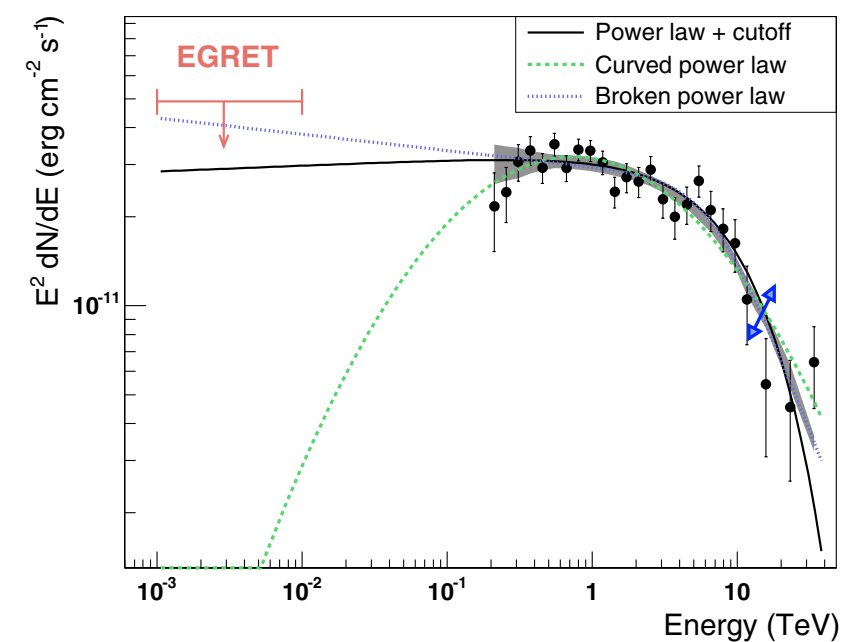

Fig. 13. HESS energy spectrum of RX J1713.7-3946. Plotted are the HESS points with their $\pm 1 \sigma$ statistical errors in an energy flux diagram. The three curves (specified in Table 1) are the best fit results of a power law with an exponential cutoff, a power law with energy dependent photon index, and a broken power law, extrapolated to $1 \mathrm{GeV}$ to enable comparisons with the EGRET upper limit in the range of 1-10 GeV. The shaded grey band represents the systematic uncertainty on the measurement, originating from the uncertainty on the background estimation. The blue arrow indicates the $20 \%$ systematic uncertainty on the energy scale, which might shift the whole curve in the given direction.

the systematic uncertainty on the background has a considerable impact on the first few flux points because of the smaller signal-to-noise ratio (as compared to points at higher energies). For the spectrum shown here the systematic uncertainty is $\approx 18 \%$ for the two lowest-energy points; it decreases rapidly with increasing energy being well below $10 \%$ at $350 \mathrm{GeV}$.

The results of the spatially resolved spectral analysis are shown in Fig. 14. It shows the distribution of photon indices over the SNR resulting from a power-law fit. The spectra were determined in rectangular regions, denoted $1-14$, each $0.26^{\circ} \times 0.26^{\circ}$ in dimension. The fit range was limited to $8 \mathrm{TeV}$ to account for (and avoid when fitting) the deviation from a power law seen in the spectrum of the whole remnant. Table 2 summarises the fit results. There is a significant flux variation over the SNR. From the brightest region in the northwest to a relatively dim one in the central part, the flux varies by more 

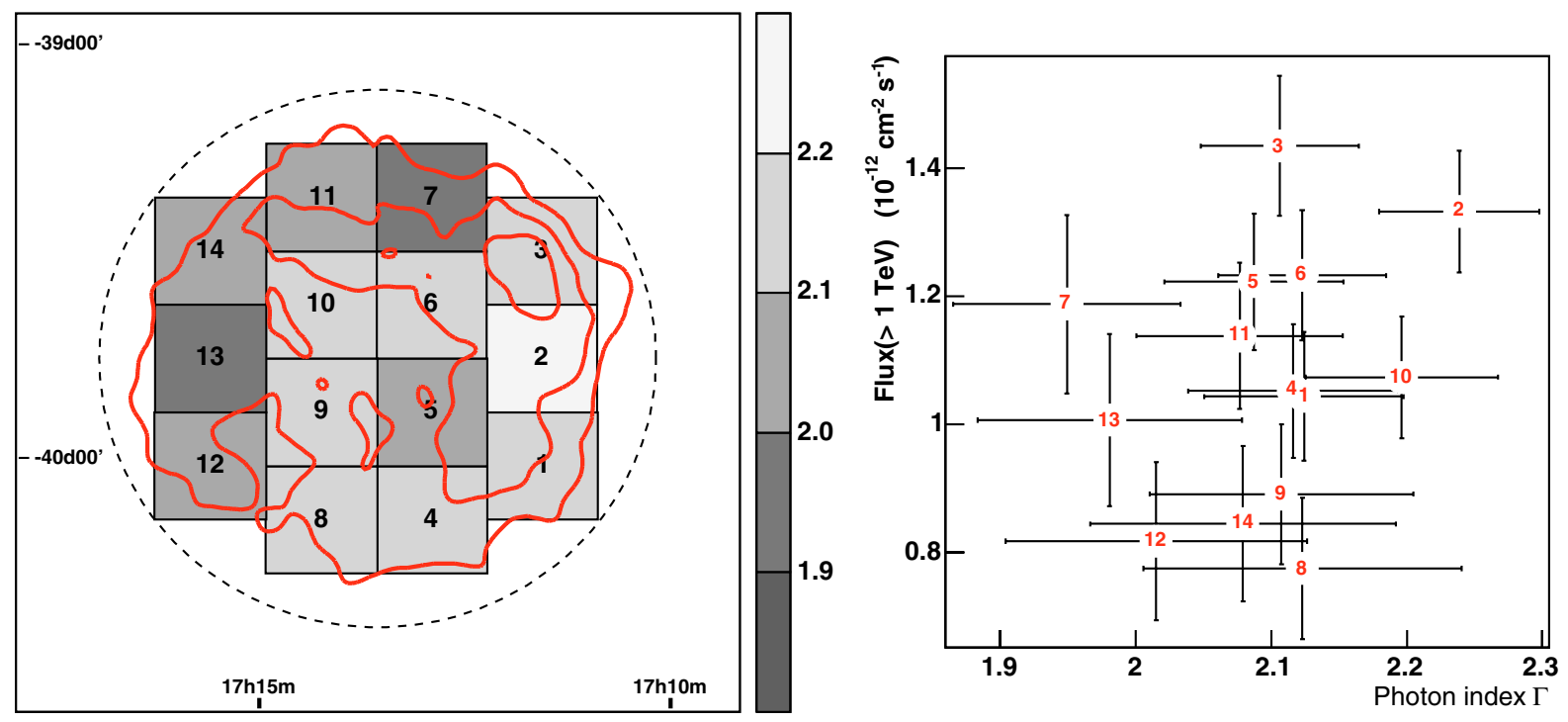

Fig. 14. The image illustrates the results of the spatially resolved spectral analysis. Left part: shown in red are gamma-ray excess contours from Fig. 7, linearly spaced at 30, 60, and 90 counts. Superimposed are the 14 boxes (each $0.26^{\circ} \times 0.26^{\circ}$ in dimension) for which spectra were obtained independently. The dashed line is the $0.65^{\circ}$ radius circle that was used to integrate events to produce a spectrum of the whole SNR. The photon index obtained from a power-law fit in each region is colour coded in bins of 0.1 . The ranges of the fits to the spectra have been restricted to a maximum of $8 \mathrm{TeV}$ (see Table 2). Right part: plotted is the integral flux above $1 \mathrm{TeV}$ against the photon index, for the 14 regions the SNR was sub-divided in. The error bars are $\pm 1 \sigma$ statistical errors. Note that systematic errors of $25 \%$ on the flux and 0.1 on the photon index are to be assigned to each data point additionally.

Table 2. Fit results for distinct regions of the SNR. Given are for each region the photon index resulting from a power-law fit, the best-fit $\chi^{2}$ and the number of degrees of freedom (d.o.f.), the integral flux above $1 \mathrm{TeV}$ and the significance of the excess events in units of standard deviation $(\sigma)$. The background for each region was determined from the same field of view, as described in Sect. 2.5.2, for each region separately, and hence the background estimates for different regions are not independent. The first row is the fit result of the whole SNR for comparison. For the whole table, the upper fit range was restricted to $8 \mathrm{TeV}$ to avoid biases due to the deviation from a power law at high energies.

\begin{tabular}{ccccc}
\hline \hline Region & $\Gamma$ & $\chi^{2}$ (d.o.f.) & $\begin{array}{c}I(>1 \mathrm{TeV}) \\
\left(10^{-12} \mathrm{~cm}^{-2} \mathrm{~s}^{-1}\right)\end{array}$ & $\begin{array}{c}\text { Excess } \\
(\sigma)\end{array}$ \\
\hline All & $2.12 \pm 0.03$ & $24.5(18)$ & $15.9 \pm 0.6$ & 30.8 \\
1 & $2.12 \pm 0.07$ & $34.7(18)$ & $1.05 \pm 0.13$ & 12.9 \\
2 & $2.24 \pm 0.06$ & $26.0(17)$ & $1.34 \pm 0.10$ & 17.2 \\
3 & $2.11 \pm 0.06$ & $30.2(18)$ & $1.45 \pm 0.13$ & 16.7 \\
4 & $2.10 \pm 0.08$ & $15.7(18)$ & $1.06 \pm 0.12$ & 11.5 \\
5 & $2.09 \pm 0.07$ & $12.6(18)$ & $1.22 \pm 0.11$ & 13.3 \\
6 & $2.13 \pm 0.06$ & $35.7(17)$ & $1.23 \pm 0.12$ & 14.1 \\
7 & $1.95 \pm 0.08$ & $9.4(16)$ & $1.19 \pm 0.12$ & 10.9 \\
8 & $2.11 \pm 0.12$ & $13.8(14)$ & $0.78 \pm 0.11$ & 8.0 \\
9 & $2.11 \pm 0.10$ & $12.5(16)$ & $0.89 \pm 0.11$ & 8.7 \\
10 & $2.19 \pm 0.07$ & $24.8(17)$ & $1.09 \pm 0.10$ & 14.1 \\
11 & $2.08 \pm 0.08$ & $11.6(15)$ & $1.13 \pm 0.11$ & 11.8 \\
12 & $2.01 \pm 0.11$ & $8.4(16)$ & $0.81 \pm 0.12$ & 8.2 \\
13 & $1.98 \pm 0.10$ & $10.7(15)$ & $1.00 \pm 0.14$ & 9.8 \\
14 & $2.08 \pm 0.11$ & $9.9(15)$ & $0.84 \pm 0.12$ & 9.4 \\
\hline
\end{tabular}

than a factor of two. There is no significant difference in spectral shape apparent, the photon indices agree with each other within statistical and systematic errors. The distribution of photon indices has a mean value of 2.09 with a root-mean-square of 0.07 . This is well compatible with the spectrum of the whole SNR when the fit range is also restricted to maximum $8 \mathrm{TeV}$ for consistency (first row in Table 2). If one adds up the integral fluxes above $1 \mathrm{TeV}$ of the individual regions, a flux of $15.1 \times$ $10^{-12} \mathrm{~cm}^{-2} \mathrm{~s}^{-1}$ is obtained, $5 \%$ less than the flux of the whole SNR (with the restriction of the fit range). This is in excellent agreement with expectations; the boxes as they are plotted in Fig. 14 cover the region of RX J1713.7-3946 with significant gamma-ray excess almost completely.

As can be seen from Fig. 14, right part, there is no correlation of the gamma-ray flux and the photon index visible in the data. This, together with the absence of any change in the spectral shape, is a remarkable difference between the gamma-ray and X-ray data. The spectral variation in X-rays was found to be much larger (see Cassam-Chenaï et al. 2004).

\section{RX J1713.7-3946 at other wavelengths}

\subsection{Comparison with $X$-rays}

There is a striking similarity between the X-ray and the gamma-ray image of RX J1713.7-3946, as they are shown in Fig. 15 for the 1-5 keV X-ray band. The overall morphology appears to be very similar, the brightest spots in both images are distributed on the shell, especially in the west. For a detailed comparison one must take into account the slightly better resolution of ASCA compared to HESS. For that purpose, the ASCA image was smoothed beyond the point-spread function of the instrument in order to match the HESS resolution. The smoothing radius was determined empirically by 


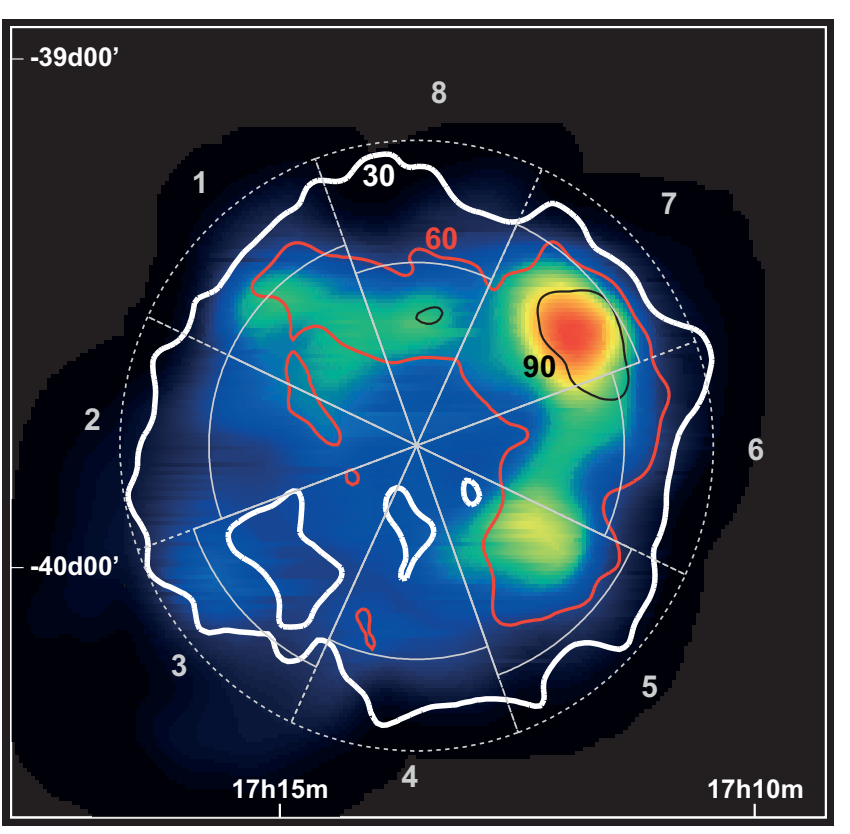

Fig. 15. ASCA X-ray (1-5 keV band, Uchiyama 2005) image of RX J1713.7-3946, overlaid with contours of the smoothed, acceptance-corrected HESS gamma-ray image. The coloured contour levels are labelled and linearly spaced at 30,60, and 90 counts. Drawn as gray thin lines are eight wedge-shaped regions for which the radial profiles are compared to each other in Fig. 16. Note that in the ASCA image, most of the regions (faint solid lines) do not reach as far as in the HESS image (faint dashed lines), accounting for the limited field of view of ASCA, whose coverage did not always extend to the boundaries of the SNR. As explained in the main text, the ASCA image was smoothed to match the HESS point-spread function to enable comparison of the two images.

smoothing the ASCA point-spread function and comparing it with the HESS point-spread function. An optimum match was obtained for a smoothing radius of $0.037^{\circ}$. Furthermore, both images in Fig. 15 are corrected for relative detector acceptance. The X-ray-bright central point source 1WGA J1713.4-3949, which was argued to be the compact relic of the supernova progenitor (see for example Cassam-Chenaï et al. 2004), was removed from the X-ray image for the purpose of comparison with gamma rays.

After degradation of ASCA's resolution, the data were compared to each other in eight wedge-shaped regions as they are drawn in Fig. 15. In each wedge, radial profiles, that is, the number of counts per unit solid angle as function of distance to the centre, were determined. To account for the differences in the absolute count level the X-ray images were scaled by a normalisation factor, which has been calculated as the ratio of $\mathrm{TeV}$ counts, integrated in a rectangle encompassing the SNR (and within the ASCA field of view), to $\mathrm{keV}$ counts, integrated in the same rectangle. The result is shown in Fig. 16 for all eight wedges. The overall good agreement in shape of the profiles is clearly visible, differences appear only at a few places, for example in region 4 and 7. For a quantitative statement on the compatibility of the two data sets, however, one would have to model and subtract the contribution from Galactic diffuse X-ray emission in the ASCA image, which amounts presumably to $10 \%$ or less in the X-ray bright parts of the SNR, but might increase to $\approx 30 \%$ in the faint parts in the east.

The interesting question of the boundaries of the SNR and if they are the same in X-rays and gamma rays can unfortunately not be addressed with the ASCA data set due to limited sky coverage.

\section{2. $C O$ and radio observations}

CO data at $2.6 \mathrm{~mm}$ wavelength of RX J1713.7-3946 and its surroundings were taken with the 4-m, mm and sub-mm telescope NANTEN in 2003 (Fukui et al. 2003). Based on positional coincidences of $\mathrm{CO}$ and $\mathrm{X}$-ray peaks and (in velocity space) shifted CO peaks, Fukui et al. (2003) concluded that the SNR blast wave is interacting with molecular clouds situated on its western side at a distance of $\approx 1 \mathrm{kpc}$. Further possible support for this scenario was recently published in Moriguchi et al. (2005), where high gas excitations are reported for this part, which could arise from heating of the molecular gas by the shock wave. The CO intensity distribution in the corresponding velocity interval is shown in Fig. 17, together with HESS gamma-ray excess contours. One notes that in the central and southeastern part of the SNR the CO emission becomes very faint or is completely absent. Apart from that, there are local $\mathrm{CO}$ maxima that coincide with $\mathrm{TeV}$-bright parts on the western side of the SNR. The azimuth profile plotted on the right-hand side of Fig. 17 illustrates a global agreement between the two measurements, regions with low gamma-ray flux reveal also low $\mathrm{CO}$ intensity, but there is no exact proportionality between the two measurements for the shell region of RX J1713.7-3946. Taking the peak values, one notes that they are shifted with respect to each other and that the gamma-ray flux varies by a factor of about three, whereas the CO intensity drops by roughly a factor of 100 in the central-southeastern part.

Figure 18 shows a comparison of the $1.4 \mathrm{GHz}$ radio image obtained with ATCA (Lazendic et al. 2004) and the HESS gamma-ray excess contours. The SNR is very faint in the radio band, there are two faint arc-like structures of emission to the west of RX J1713.7-3946, almost perpendicular to each other, one of them directly coincident with the brightest $\mathrm{TeV}$ emission region. There is no notable resemblance between the two wavelength regimes. Spectral analysis of the X-ray- and TeV-bright northwestern part of the SNR shell yields a spectral index of $0.50 \pm 0.40$, derived from two flux values taken at $1.4 \mathrm{GHz}$ and $2.5 \mathrm{GHz}$. This measurement is used further down when comparing the HESS spectral data to broadband models.

\section{Possible emission processes}

One of the key issues in the interpretation of the observed gamma-ray emission is the identification of the particle population responsible for the generation of the gamma rays. The close correlation between X-rays and gamma rays might indicate an electronic origin; models of supernova remnants as Galactic cosmic-ray sources, on the other hand, suggest that primarily a hadronic component from pion decays exists. 


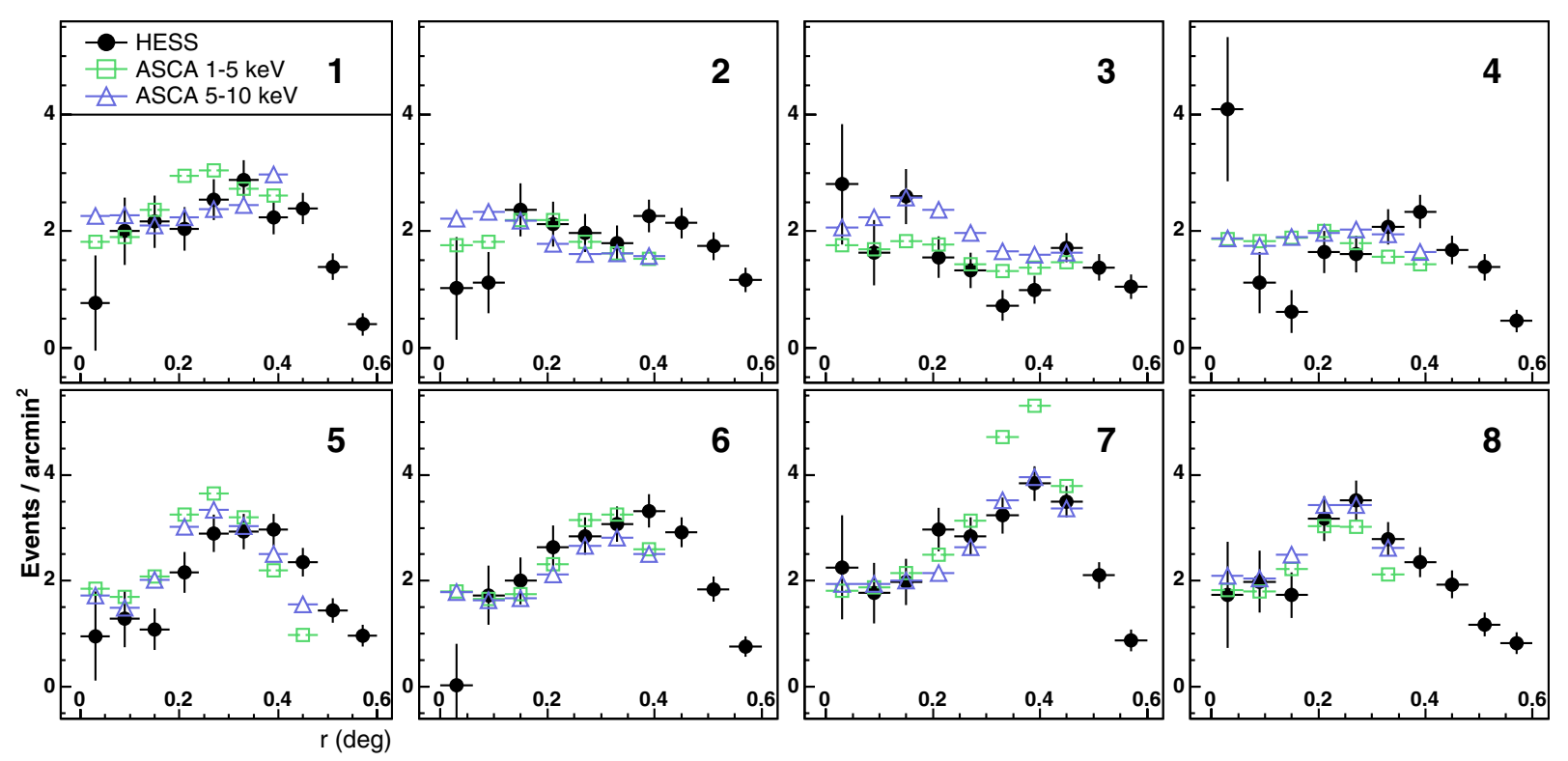

Fig. 16. Radial profiles for the eight regions marked in Fig. 15. Plotted are HESS excess counts per unit solid angle (solid circles) as a function of distance $r$ in degrees to the centre of the SNR, compared to soft (1-5 keV) and hard (5-10 keV) X-ray data. All data were corrected for relative acceptance. There is a very good general agreement between the $\mathrm{keV}$ and $\mathrm{TeV}$ data sets. The most pronounced differences appear in regions 4 , where the $\mathrm{TeV}$ flux drops almost to zero at $\approx 0.15^{\circ}$ from the centre, and 7 , where a pronounced peak appears in the $1-5 \mathrm{keV} \mathrm{X}$-ray data, which repeats neither in the 5-10 keV X-ray nor the TeV data. Note that the X-ray emission includes diffuse Galactic X-ray emission.
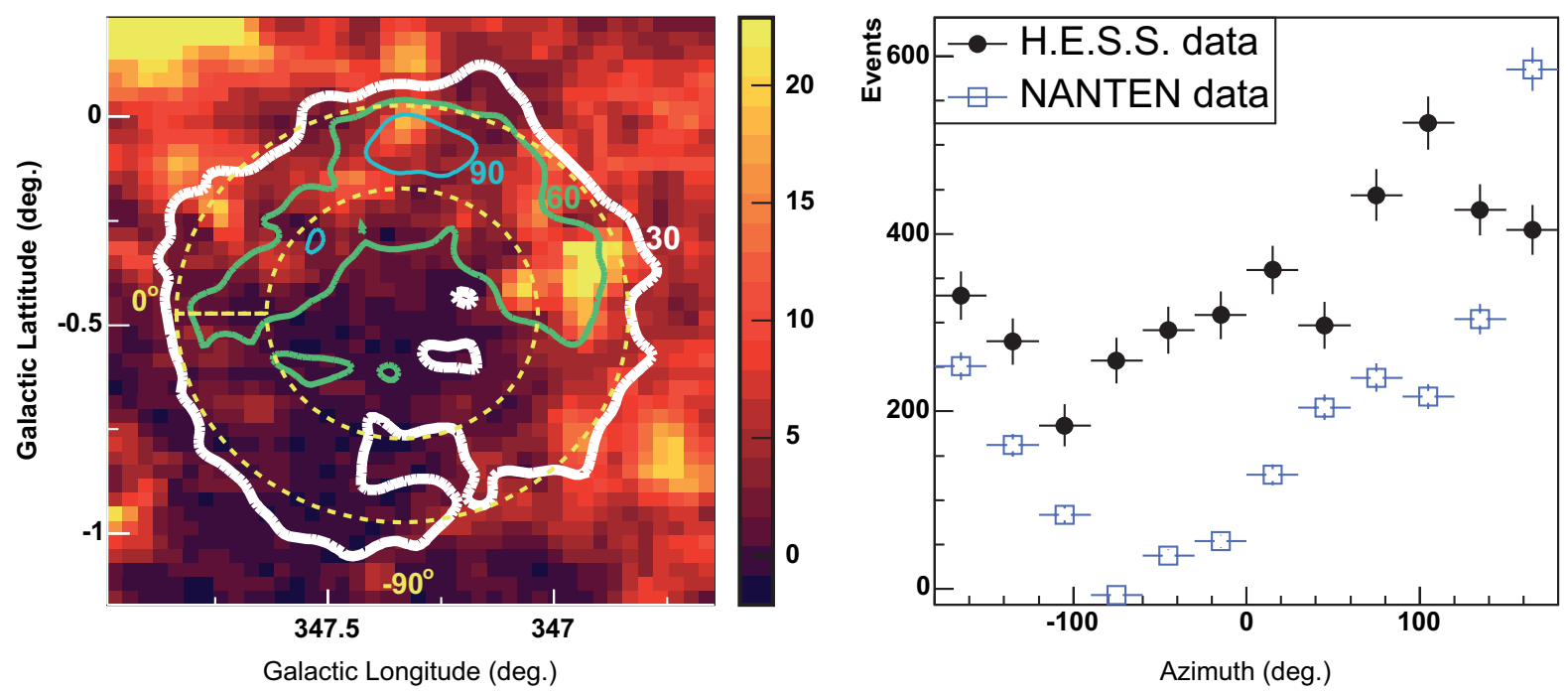

Fig. 17. Left panel: shown are the intensity distribution of $\mathrm{CO}(J=1-0)$ emission (Fukui et al. 2003) (linear colour scale in units of $\mathrm{K} \mathrm{km} \mathrm{s}^{-1}$, truncated at a value of 23 to highlight important features), derived by integrating the CO spectra in the velocity range from $-11 \mathrm{~km} \mathrm{~s}{ }^{-1}$ to $-3 \mathrm{~km} \mathrm{~s}^{-1}$ (which corresponds to $0.4 \mathrm{kpc}$ to $1.5 \mathrm{kpc}$ in space). Overlaid are coloured contours of the HESS gamma-ray excess image. The levels are labelled and linearly spaced at 30,60, and 90 counts. Note that the image is shown in Galactic coordinates. Right panel: azimuth profile plot, that is, number of counts as a function of the azimuthal angle, integrated in a $0.2^{\circ}$-wide ring covering the shell of RX J1713.7-3946 (dashed yellow circle in the left-hand panel). Plotted are the HESS gamma-ray and the NANTEN data set.

To identify the different contributions, the wide-band electromagnetic spectra from radio to multi-TeV gamma-rays must be compared to model calculations.

In the literature, different schemes are employed to model broadband emission from SNRs. Phenomenologically oriented models (Mastichiadis \& de Jager 1996; Aharonian \& Atoyan 1999) start by ad hoc assuming particle acceleration spectra usually as power laws with a cutoff - to derive particle spectra taking into account energy losses and then calculate the electromagnetic spectrum with additional assumptions concerning the local magnetic field, the radiation fields which serve as target for the IC process, and the gas density. Spectral parameters are either taken from acceleration models, resulting in a spectral index around 2, or determined from data. More sophisticated gamma-ray models account for the non-linear effects arising from the interaction of the accelerated particles 


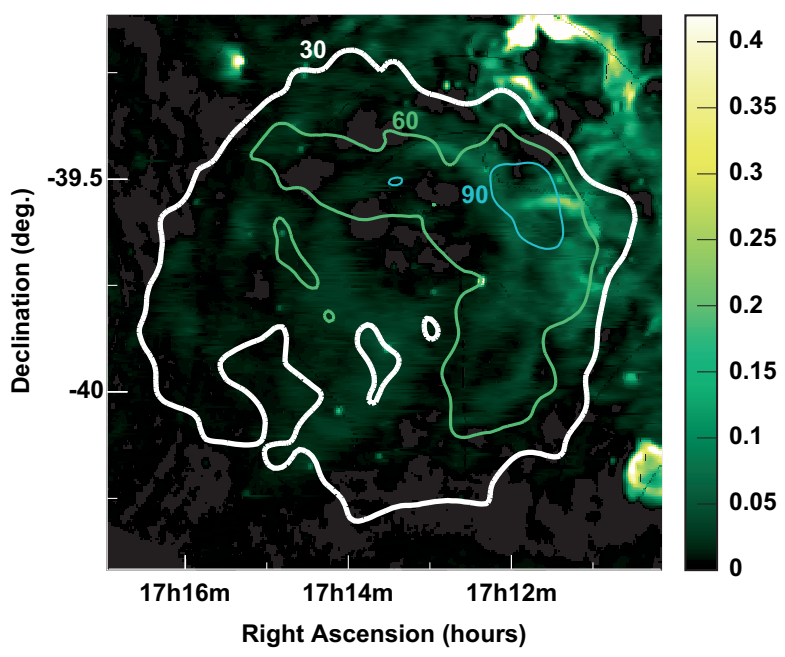

Fig. 18. 1.4 GHz ATCA radio image (Lazendic et al. 2004, courtesy of P. Slane). The linear colour scale is in units of Jy beam ${ }^{-1}$. Overlaid are coloured contour lines of the HESS gamma-ray excess image.

with the shocked supernova shell, which result in deviations from pure power laws, with spectra flattening at higher energies (Berezhko \& Völk 1997; Baring et al. 1999).

The original discovery paper of VHE gamma-ray emission from RX J1713.7-3946 (Muraishi et al. 2000) claimed electrons as the likely source particle population. However, it soon became evident that a consistent modelling of the spectra is hard to achieve in simple one-zone models. Apart from the choice of the electron spectrum, the only free parameter is the magnetic field strength, which controls the spacing of the synchrotron and IC peaks in the SED together with their relative intensities and - one should add - the amount of radiative cooling of the accelerated component. Enomoto et al. (2002) noted that for modest magnetic fields $-B$ equal to a few $\mu \mathrm{G}-$ the measured intensity ratios are reproduced but the gammaray spectra are much too hard. Using higher fields, one can match the gamma-ray spectra at the expense of dramatically increased X-ray yields. While the HESS data differ from the CANGAROO-II data both in terms of the region covered and the exact values for flux and index, this conclusion for the electronic scenario remains basically valid. The agreement can be improved by introducing an additional parameter to decouple the X-ray intensity and the spectral shape, namely the magnetic field filling factor which allows the X-ray flux to be tuned without change of the spectra. With very small filling factors of 0.001 (Pannuti et al. 2003) to 0.01 (Lazendic et al. 2004), difficult to justify physically, the X-ray and the CANGAROO-II gamma-ray spectra can be described for magnetic fields around $10 \mu \mathrm{G}$ to $15 \mu \mathrm{G}$ in the emitting region. This latter approach is not followed here.

The validity of electronic models could be judged more easily if the magnetic field values in the remnant were known. For typical shock compression ratios around 4 and pre-shock interstellar fields of a few $\mu \mathrm{G}$, fields of $10 \mu \mathrm{G}$ to $15 \mu \mathrm{G}$ are at the lower limit of the expected range; mechanisms of dynamical field amplification in non-linear shocks (Lucek \& Bell 2000; Bell \& Lucek 2001; Bell 2004) will generally result in higher fields. The narrow filaments visible in many high-resolution X-ray images of SNRs (see, e.g., Bamba et al. 2005) have been pointed out to provide means to probe magnetic fields (Vink \& Laming 2003; Berezhko et al. 2003): only relatively high fields can result in sufficiently rapid cooling of electrons to make such filamentary features possible and visible. On the basis of the structures seen in Chandra images in the northwest of RX J1713.7-3946 (Uchiyama et al. 2003), Völk et al. (2005) have argued that fields between $58 \mu \mathrm{G}$ and a few $100 \mu \mathrm{G}$ might be possible, depending on the detailed assumptions about the remnant's morphology ${ }^{1}$. Such high fields - likely to be present throughout the remnant - would rule out a leptonic origin of VHE gamma rays right away.

On the basis of the difficulty of accommodating broadband spectra in a single-zone electronic model, Enomoto et al. (2002) proposed RX J1713.7-3946 as the first wellidentified proton accelerator. This interpretation was criticised by Butt et al. (2002) and Reimer \& Pohl (2002) since the CANGAROO-II spectra, extrapolated to lower energies, would violate the flux level of the nearby EGRET source 3EG 1714-3857 (Hartman et al. 1999), which, if not associated with RX J1713.7-3946, must then be considered as upper limit on the $\mathrm{GeV}$ emission. However, the EGRET limit can be circumvented by reducing the amount of low-energy protons compared to the $E^{-2}$ spectrum. This can be achieved by the ad hoc assumption of a spectral break, or - for the CANGAROO-II data - by assuming a flatter overall spectrum with a photon index smaller than 2 .

A very detailed modelling is beyond the scope of this paper; the models presented in the following serve mainly to illustrate that spectra and energetics can be reproduced with plausible input parameters.

\subsection{Electronic scenario}

In Fig. 19 the synchrotron and IC emission from relativistic electrons are modelled within the framework of a one-zone model in which the electron acceleration and gamma-ray emission take place in the same region. It is assumed that the primary electrons follow a power law with index $\alpha=2$ and with an exponential cutoff $E_{0}$,

$Q(E)=Q_{0} E^{-\alpha} \exp \left(-E / E_{0}\right)$,

and that they are produced continuously over a fixed time interval $T$ inside a region with given homogeneous distributions of magnetic field strength $B$ and ambient gas density $n$. The energy distribution of the electrons is then calculated taking into account energy losses due to IC and synchrotron emission, Bremsstrahlung and ionisation as well as losses due to Bohm diffusion. The broadband energy distribution of the source is calculated for an age of $T=1000$ years, an average gas density of $n=1 \mathrm{~cm}^{-3}$, and a distance to the source of $D=1 \mathrm{kpc}$. For the IC emission, canonical interstellar values for the seed photon densities were considered: $W_{\mathrm{CMB}}=0.25 \mathrm{eV} \mathrm{cm}^{-3}$ for the

1 We note that in Hiraga et al. (2005), Fig. 2, a radial profile from the XMM image is shown. It reveals another very thin filament-like structure in the west of RX J1713.7-3946 which is a sign of high magnetic field values. 


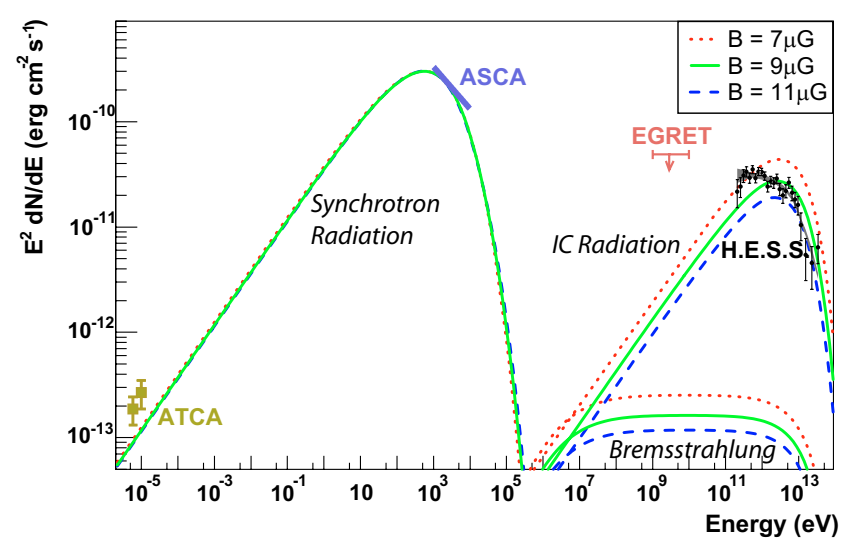

Fig. 19. Broadband SED of RX J1713.7-3946. The ATCA radio data and ASCA X-ray data (Hiraga 2005) for the whole SNR are indicated, along with the HESS measurement and the EGRET upper limit. Note that the radio flux was determined in Lazendic et al. (2004) for the northwest part of the shell only and was scaled up by a factor of two here to account for the whole SNR. The synchrotron and IC spectra were modelled assuming a source distance of $1 \mathrm{kpc}$, an age $T$ of 1000 years, a density $n$ of $1 \mathrm{~cm}^{-3}$, and a production rate of relativistic electrons by the acceleration mechanism in the form of a power law of index $\alpha=2$ and an exponential cutoff of $E_{0}=100 \mathrm{TeV}$. Shown are three curves for three values of the mean magnetic field: $7 \mu \mathrm{G}$, $9 \mu \mathrm{G}$, and $11 \mu \mathrm{G}$, to demonstrate the required range of the $\mathrm{B}$ field strength for this scenario. The electron luminosity is adopted such that the observed X-ray flux level is well matched. For the three magnetic field values the luminosity $L_{\mathrm{e}}$ is $L_{\mathrm{e}}=1.77 \times 10^{37} \mathrm{erg} \mathrm{s}^{-1}(7 \mu \mathrm{G})$, $L_{\mathrm{e}}=1.14 \times 10^{37} \mathrm{erg} \mathrm{s}^{-1}(9 \mu \mathrm{G})$, and $L_{\mathrm{e}}=0.81 \times 10^{37} \mathrm{erg} \mathrm{s}^{-1}(11 \mu \mathrm{G})$.

cosmic microwave background (CMB), $W_{\mathrm{SL}}=0.5 \mathrm{eV} \mathrm{cm}^{-3}$ for optical star light and $W_{\mathrm{IR}}=0.05 \mathrm{eV} \mathrm{cm}^{-3}$ for infrared background light. The absolute electron production rate, $Q_{0}$, is determined from the constraint of matching the observed X-ray flux level. Figure 19 shows the resulting model curves, together with measurements in various wavelength regimes, for three different average magnetic field values. From the absolute levels it is evident that a magnetic field around $10 \mu \mathrm{G}$ is required in order to explain both the X-ray and gamma-ray flux levels. On the other hand one notes that such a model with the above mentioned parameters does not provide a reasonable description of the HESS data. The IC peak appears too narrow to reproduce the flat $\mathrm{TeV}$ emission. The detailed inclusion of non-linear acceleration effects should not change the situation very much. They are expected to steepen the synchrotron SED above the radio range. Synchrotron cooling of the accelerated electrons then tends to produce a flat-topped synchrotron and accordingly IC maximum. It is, however, a long way to flatten the IC spectrum so extensively at low energies as to achieve agreement with the HESS spectrum.

Obviously, the simple model presented here served basically to underline the main arguments. Nevertheless, the conclusion that a power-law production spectrum fails to simultaneously account for the radio, X-ray and gamma-ray data appears to be a generic and stable feature; additional parameters are required to decouple either the $\mathrm{TeV}$ and $\mathrm{X}$-ray/radio fluxes - such as a fillingfactor - or the X-ray and radio

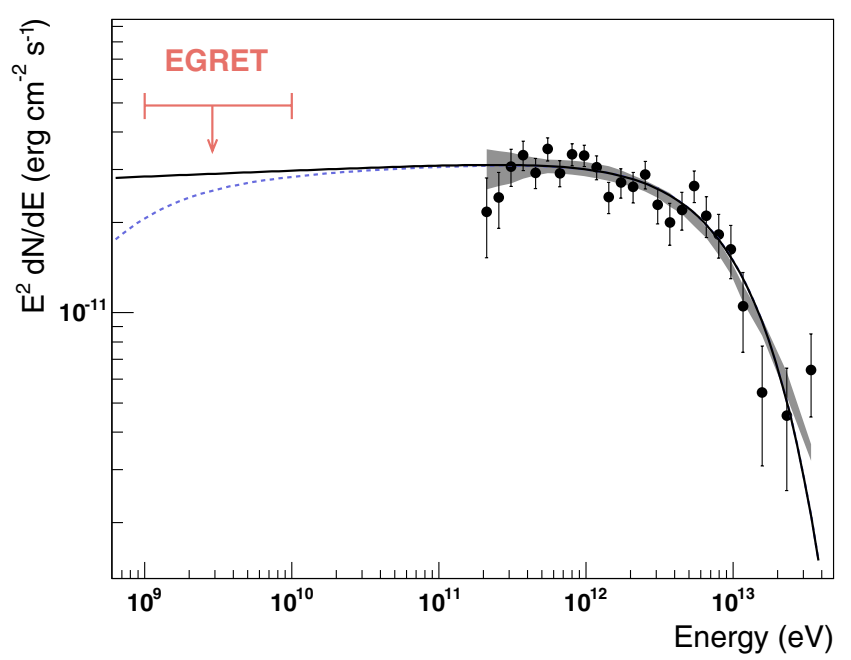

Fig. 20. HESS data points plotted in an energy flux diagram. The shaded grey band is the systematic error band for this measurement (see Sect. 3.2). The black curve is the best fit of a power law with exponential cutoff to the data, extrapolated to lower energies. The dashed blue curves is the same function, but it takes the $\pi^{0}$ kinematics into account. The EGRET upper limit from $1 \mathrm{GeV}$ to $10 \mathrm{GeV}$ is plotted as red arrow.

spectra - such as an ad-hoc spectral break, which for the given source age and magnetic field can not be justified as an effect of radiative cooling.

\subsection{Hadronic scenario}

Assuming alternatively that nuclear cosmic-ray particles, accelerated at the SNR shock, dominantly produce VHE gamma rays, theoretically the most plausible differential energy spectrum of accelerated nuclei is a concave $E^{-\Gamma(E)}$-type spectrum, due to nonlinear back coupling, with a cutoff at gamma-ray energy $E_{\mathrm{c}}$, where $\Gamma$ is decreasing towards higher energies (just below the $\mathrm{TeV}$ energy range) to a value between 1.5 and 2 , before the spectrum is steepening again in the cutoff region. In the test-particle approximation one expects $\Gamma \simeq 2$. The HESS spectrum is indeed compatible with such a scenario. Figure 20 shows a $v F_{v}$ representation of the HESS data, together with the best-fit curve of a power law with an exponential cutoff (see Sect. 3.2, Table 1), extrapolated to small energies. Compared to that a curve is plotted which takes the kinematics of the production process of gamma rays, $p p \rightarrow \pi^{0} \rightarrow \gamma \gamma$, into account. The power law spectrum continues to smaller energies with an index of $\approx 2$, as expected in the test-particle limit, until the suppression of gamma rays due to $\pi^{0}$-decay kinematics is encountered and the curve is turning down. Note that already the extrapolation of the HESS spectrum is well below the EGRET upper limit from the position of RX J1713.7-3946, introduced in Sect. 3.2. Taking into account non-linear effects would harden the gamma-ray spectrum even more.

One should mention at this point that on the theory side other mechanisms to suppress contributions from lowenergy ( $\mathrm{E}$ around $10 \mathrm{GeV}$ ) protons have been considered 
(e.g., Malkov et al. 2005). When the particles upstream of the shock hit a dense target with a spatial gradient, such as a dense molecular cloud, the gamma-ray emission of low-energy protons might also be suppressed due to the energy dependence of the diffusion length. In a more general context, such mechanisms - an accelerator of finite lifetime interacting with a target at a distance where diffusion time scales are comparable to the source lifetime - have been studied by Aharonian \& Atoyan (1996). Such arguments, however, need to be reassessed when the exact location of the clouds with respect to the shock front is known.

To calculate the energetics in a hadronic scenario, the mean target gas density available for gamma-ray production in the region of RX J1713.7-3946 is a key question. The CO image shown in Fig. 17, left-hand side, reveals a hole in the $\mathrm{CO}$ emission and accordingly in the molecular hydrogen distribution in the central and eastern part of the SNR. In contrast, the TeV emission fills the whole region of the SNR (see Fig. 7). As is shown on the right-hand side of Fig. 17, there is no exact correlation between VHE gamma rays and CO intensity. From this one can conclude that in all likelihood cosmic rays do not penetrate the clouds uniformly. The bulk of the VHE gamma rays is not linked to the molecular clouds but must be due to interactions with a different target. Indeed, the rather good spherical shape of the remnant together with the fact that the X-ray and gamma-ray emission only varies by a factor of two to four across the remnant lends further support to the scenario that the SNR is running into a more or less uniform and probably low-density environment. Although it seems to be beginning to interact with the dense clouds to the west, the ones that are seen by NANTEN, VHE gamma rays are dominantly produced in cosmic-ray interactions with rather uniform ambient gas. One possible scenario is that the SNR is the result of a core-collapse supernova explosion that occurred into a wind bubble of a massive progenitor star. An SNR shock expanding into the bubble, with an ambient density much lower than that suggested by an average molecular cloud scenario, could explain the relative uniformity of the gamma-ray emission, compared to the large density variations in the clouds which likely surround the remnant (for a theoretical treatment of such configurations, see Berezhko \& Völk 2000).

The local target density is a crucial parameter in this scenario. With the NANTEN measurement of the void in the central part of the SNR one might constrain the local density in that region. The sensitivity of the final NANTEN data set as quoted in Fukui et al. (2003) corresponds to a molecular column density of $8.3 \times 10^{19} \mathrm{~cm}^{-2}$ assuming the conventional conversion relation from $\mathrm{CO}$ intensity to $\mathrm{H}_{2}$ column density $\left(0.4 \mathrm{~K} \mathrm{~km} \mathrm{~s}^{-1}\right)$. Taking the diameter of the SNR as $\approx 20 \mathrm{pc}$ for $1 \mathrm{kpc}$ distance, one can deduce an upper limit on the molecular hydrogen column density of $\approx 2.6 \mathrm{~cm}^{-3}$ in parts of the SNR without detectable $\mathrm{CO}$ emission. The other existing constraint was inferred in Cassam-Chenaï et al. (2004) from XMM data, based on the lack of thermal X-ray emission. By fitting the spectra with an absorbed power-law model and adding a thermal component, an upper limit on the mean gas temperature and, important here, the mean hydrogen number density of the ambient pre-shock medium of $0.02 \mathrm{~cm}^{-3}$ was obtained.
One should note, though, that this value is likely to be too low if the shocks are strongly modified by the accelerated particles, the shock heating is substantially reduced and the data would be consistent with higher densities.

Assuming for now a mean target gas density of $n \approx 1 \mathrm{~cm}^{-3}$, uniformly spread throughout the remnant, in accordance with the NANTEN, but not the XMM limit, one can calculate the proton energetics implied by the gamma-ray flux measured from 0.2 to $40 \mathrm{TeV}$. The total energy in accelerated protons from about $2-400 \mathrm{TeV}$, required to provide the observed flux, can be estimated as $W_{p}^{\mathrm{tot}}(2-400 \mathrm{TeV}) \approx t_{p p \rightarrow \pi^{0}} \times$ $L_{\gamma}(0.2-40 \mathrm{TeV})$, where $t_{p p \rightarrow \pi^{0}} \approx 4.5 \times 10^{15}\left(n / 1 \mathrm{~cm}^{-3}\right)^{-1} \mathrm{~s}$ is the characteristic cooling time of protons through the $\pi^{0}$ production channel, $L_{\gamma}(0.2-40 \mathrm{TeV})=4 \pi d^{2} w_{\gamma}(0.2-40 \mathrm{TeV})$ is the luminosity of the source in gamma rays between 0.2 and $40 \mathrm{TeV}$, and $w_{\gamma}(0.2-40 \mathrm{TeV})$ is the gamma-ray energy flux for the corresponding energy range. Assuming then that the proton spectrum with spectral index $\alpha \approx \Gamma$ continues down to $1 \mathrm{GeV}$, the total energy in protons can be estimated and compared to the total assumed mechanical explosion energy of the supernova of $10^{51} \mathrm{erg}$. These calculations reveal very similar values for the three spectral shapes given in Fig. 20 in the gamma-ray energy range between 0.2 and $40 \mathrm{TeV}$ : for the gamma-ray energy flux one obtains $w_{\gamma}(0.2-40 \mathrm{TeV}) \approx 10^{-10} \mathrm{erg} \mathrm{cm}^{-2} \mathrm{~s}^{-1}$, the gamma-ray luminosity is $L_{\gamma}(0.2-40 \mathrm{TeV}) \approx 10^{34}\left(\frac{d}{1000 \mathrm{pc}}\right)^{2} \mathrm{erg} \mathrm{s}^{-1}$, and the corresponding energy content of protons is $W_{p}^{\mathrm{tot}}(2-400 \mathrm{TeV}) \approx$ $6 \times 10^{49}\left(\frac{d}{1000 \mathrm{pc}}\right)^{2}\left(\frac{n}{1 \mathrm{~cm}^{-3}}\right)^{-1}$ erg. The resulting total energy in protons, after extrapolating the proton spectrum to $1 \mathrm{GeV}$ and using $E_{51} \equiv 10^{51}\left(\frac{d}{1000 \mathrm{pc}}\right)^{2}\left(\frac{n}{1 \mathrm{~cm}^{-3}}\right)^{-1} \mathrm{erg}$, is then $W_{p}^{\text {tot }} \approx 0.19 \times E_{51}$ for a power law with exponential cutoff, $W_{p}^{\text {tot }} \approx 0.08 \times E_{51}$ for a power law with energy dependent index, and $W_{p}^{\text {tot }} \approx 0.26 \times E_{51}$ for a broken power law. These numbers are consistent with the notion of an SNR origin of Galactic cosmic rays involving the canonical $\approx 10 \%$ conversion efficiency of the total supernova explosion energy. The HESS gamma-ray flux level is close to what was predicted in Drury et al. (1994) from nearby young SNRs for ambient densities of $n \approx 1 \mathrm{~cm}^{-3}$. One should keep in mind though that the order of magnitude uncertainties in the measurements of the distance to the source $d$ and of the local gas density $n$ feed directly into these estimates.

\subsection{Discussion and conclusions}

The models and ideas presented in this section were aiming at exploring the possibilities available in explaining the observed VHE emission in purely electronic and purely hadronic scenarios. It is found that in the hadronic scenario, assuming gamma rays to stem from $\pi^{0}$ decays, the extrapolation of the HESS spectrum to lower gamma-ray energies leads to a picture that is consistent with the low-energy EGRET data. Furthermore, the spectral shape is well compatible with cosmic-ray acceleration theory. The energy requirements implied by the gamma-ray flux are in agreement with expectations from cosmic-ray acceleration in shell-type SNRs 
in our Galaxy, if one assumes a local target gas density of $n \approx 1 \mathrm{~cm}^{-3}$ and takes the currently preferred distance estimate of $1 \mathrm{kpc}$. Unfortunately both of these parameters are not very well measured. The distance estimate, which factors quadratically into the energetics calculation, has uncertainties in the order of at least $30 \%$. For the local target density there exist only upper limits, since from comparisons with $\mathrm{CO}$ data it turns out that gamma rays are most likely not exclusively linked to the dense molecular clouds surrounding the SNR. These clouds, however, obscure the measurement of the actual local target material available for gamma-ray production. Only towards the interior and the southeast of the SNR, where there is a hole in the molecular column density, is there hope to actually measure and constrain the density. Existing estimates in these regions are the NANTEN upper limit of $2.6 \mathrm{~cm}^{-3}$, which does not cause any problem with the assumption made above, and the XMM upper limit of $0.02 \mathrm{~cm}^{-3}$ which, if correct, would seriously challenge the idea of a hadronic scenario of gamma-ray production at least for this object.

In the electronic scenario, on the other hand, the data are not easily reproduced taking only IC emission into account. The very low magnetic field of $\approx 10 \mu \mathrm{G}$, fixed by the ratio of synchrotron to IC flux, exceeds typical interstellar values only slightly and is difficult to reconcile with the paradigm of the diffusive shock acceleration of cosmic rays at supernova shock waves which predicts strong field amplifications in the region of the shock (Lucek \& Bell 2000; Bell \& Lucek 2001; Bell 2004). In the case of RX J1713.7-3946 it was indeed considered possible by Völk et al. (2005) that the magnetic field strength at the SNR shock front significantly exceeds typical interstellar values.

Complete understanding of gamma-ray emission processes can only be achieved by taking a broadband approach and using all the available measurements in the different wavelength regimes. In Sect. 4 the TeV data set was compared to X-ray, radio and $\mathrm{CO}$ emission measurements of the region surrounding RX J1713.7-3946. While there is no obvious resemblance with the radio image, it turns out that there is a striking spatial correlation between the ASCA X-ray and the HESS gamma-ray data. Most of the emission regions seem to exhibit exactly the same morphology in both wavelength regimes. At first sight this supports the idea that X-rays and gamma rays are produced by the same particle population, namely electrons. Assuming a constant magnetic field throughout the remnant (not the most likely configuration), the intensity (and spectrum) of both synchrotron and IC radiation trace the density (and the spectrum) of electrons, giving rise to the observed correlation. If the VHE gamma rays were due to non-thermal Bremsstrahlung of electrons, which is correlated with gas density, the observed correlation could be due to a magnetic field and gas density correlation. However, as can be seen from Fig. 19, Bremsstrahlung dominates over IC radiation only for very large values of $n_{\mathrm{H}}>100 \mathrm{~cm}^{-3}$, which are not compatible with the CO measurements from the centre of the SNR, as mentioned above. But even given such a high density it is questionable if density, field strength and electron spectra can be fine-tuned such that the experimental results are approximately reproduced. Another difficulty for an electronic interpretation arises from the observation by Cassam-Chenaï et al. (2004) that the X-ray spectra are steeper in the presumed shock front in the west, where the blast wave probably impacts the molecular cloud, than in the southeast, where the front propagates into a low density medium. It is very difficult to explain why the spectral shape in X-rays, but not in gamma rays, changes significantly in distinct regions of the shock, if they stem from the same particle population. If on the other hand gamma rays originate dominantly from nucleonic cosmic rays, a spatial correlation between X-rays and gamma rays is not automatically ensured either. There are two possible scenarios. The correlation could point to a common acceleration process accelerating both electrons and protons - indeed expected in the theory of diffusive shock acceleration - such that the spatial distributions are to first order the same and only differ because of the different loss processes. The second alternative is a correlated enhancement of magnetic field and local gas density.

Another possibility of course is that the VHE gamma rays are a roughly equal mixture of two components, produced by both electrons and protons. However, this scenario seems unlikely since the energy-independent gamma-ray morphology and the absence of variations in spectral shape would again require fine-tuning of parameters like the magnetic field $B$ and the ambient density $n$.

We conclude that the straightforward and simplest approaches in both scenarios lead to problems and one has difficulties in finding unequivocal evidence for either of them when using all the available broadband data. Nevertheless, the shape of the gamma-ray spectrum favours a hadronic scenario.

\section{Summary}

The VHE gamma-ray emission of RX J1713.7-3946 was measured with unprecedented precision with HESS. The accuracy of the measurement is now approaching the level of X-ray measurements of this source, with the distinct advantage that HESS covers the whole SNR within its field of view. With the 2004 data, a close spatial correlation between X-rays and gamma rays was found. The overall gamma-ray energy spectrum was measured over more than two decades. There are indications for a deviation from a pure power-law spectrum. The data seem to be reasonably well described by a power law with an exponential cutoff and a power law with energy dependent photon index, as well as a broken power law. At the current stage further investigations about the shape of the spectrum at the highest energies accessible to HESS are hampered by the limited event statistics. The large data set has allowed for a spatially resolved spectral study. No significant variation in the gamma-ray spectral shape over the SNR is found. The flux varies by more than a factor of two across the SNR. The northern and western parts of the shell, where the SNR is believed to impact molecular clouds, are significantly brighter than the remaining parts.

We presented broadband modelling ideas and discussed RX J1713.7-3946 in terms of the available data from all wavelength bands including the HESS gamma-ray signal. Two scenarios were addressed, one where gamma rays originate from electrons and one where they originate from protons. In both 
cases the large uncertainties on crucial parameters like the magnetic field strength and the effective ambient density, which are not directly accessible to measurements, hamper decisive conclusions. Nevertheless, the proton scenario is favoured because of the shape of the gamma-ray spectrum. From the theory side, the remaining challenge is the connection of the different particle species, VHE electrons and nuclei, in a consistent broadband model of RX J1713.7-3946. Experimentally, with the current gamma-ray data set, more precise measurements of the surrounding molecular clouds are clearly needed in order to link emission regions of VHE gamma rays to regions of known density.

Acknowledgements. The support of the Namibian authorities and of the University of Namibia in facilitating the construction and operation of HESS is gratefully acknowledged, as is the support by the German Ministry for Education and Research (BMBF), the Max Planck Society, the French Ministry for Research, the CNRS-IN2P3 and the Astroparticle Interdisciplinary Programme of the CNRS, the U.K. Particle Physics and Astronomy Research Council (PPARC), the IPNP of the Charles University, the South African Department of Science and Technology and National Research Foundation, and by the University of Namibia. We appreciate the excellent work of the technical support staff in Berlin, Durham, Hamburg, Heidelberg, Palaiseau, Paris, Saclay, and in Namibia in the construction and operation of the equipment. We also thank Y. Uchiyama for supplying the ASCA X-ray data and assisting with the comparisons with the HESS data, and Y. Moriguchi and Y. Fukui for supplying the NANTEN data.

\section{References}

Aharonian, F. A., \& Atoyan, A. M. 1996, A\&A, 309, 917

Aharonian, F. A., \& Atoyan, A. M. 1999, A\&A, 351, 330

Aharonian, F. A., Drury, L. O., \& Voelk, H. J. 1994, A\&A, 285, 645

Aharonian, F. A., Akhperjanian, A. G., Aye, K.-M., et al. (HESS Collaboration) 2004a, APh, 22, 109

Aharonian, F. A., Akhperjanian, A. G., Aye, K.-M., et al. (HESS Collaboration) 2004b, Nature, 432, 75

Aharonian, F. A., Akhperjanian, A. G., Bazer-Bachi, A. R., et al. (HESS Collaboration) 2005a, A\&A, 437, L7

Aharonian, F. A., Akhperjanian, A. G., Aye, K.-M., et al. (HESS Collaboration) 2005b, A\&A, 430, 865

Aharonian, F. A., Akhperjanian, A. G., Aye, K.-M., et al. (HESS Collaboration) 2005c, in preparation

Aharonian, F. A., Akhperjanian, A. G., Aye, K.-M., et al. (HESS Collaboration) 2006, ApJ, 636, 777

Aschenbach, B. 1998, Nature, 396, 141

Atkins, R., Benbow, W., Berley, D., et al. 2004, ApJ, 608, 680

Bamba, A., Yamazaki, R., Yoshida, T., Terasawa, T., \& Koyama, K. 2005, ApJ, 621, 793

Baring, M. G., Ellison, D. C., Reynolds, S. P., Grenier, I. A., \& Goret, P. 1999, ApJ, 513, 311

Bell, A. R. 2004, MNRAS, 353, 550

Bell, A. R., \& Lucek, S. G. 2001, MNRAS, 321, 433

Berezhko, E. G., \& Völk, H. J. 1997, APh, 7, 183

Berezhko, E. G., \& Völk, H. J. 2000, A\&A, 357, 283

Berezhko, E. G., Ksenofontov, L. T., \& Völk, H. J. 2003, A\&A, 412, L11
Bernlöhr, K., Carrol, O., Cornils, R., et al. 2003, APh, 20, 111

Blandford, R., \& Eichler, D. 1987, Phys. Rep., 154, 1

Butt, Y. M., Torres, D. F., Romero, G. E., Dame, T. M., \& Combi, J. A. 2002, Nature, 418, 499

Cassam-Chenaï, G., Decourchelle, A., Ballet, J., et al. 2004, A\&A, 427, 199

Cornils, R., Gillessen, S., Jung, I., et al. 2003, APh, 20, 129

Drury, L. O., Aharonian, F. A., \& Völk, H. J. 1994, A\&A, 287, 959

Ellison, D. C., Slane, P., \& Gaensler, B. M. 2001, ApJ, 563, 191

Enomoto, R., Tanimori, T., Naito, T., et al. 2002, Nature, 416, 823

Fukui, Y., Moriguchi, Y., Tamura, K., et al. 2003, PASJ, 55, L61

Funk, S., Hermann, G., Hinton, J., et al. 2004, APh, 22, 285

Ginzburg, V. L., \& Syrovatskii, S. I. 1964, The Origin of Cosmic Rays (New York: Macmillan)

Hartman, R. C., Bertsch, D. L., Bloom, S. D., et al. 1999, ApJS, 123, 79

Hillas, A. M. 1985, in Proc. 19th ICRC, 445

Hinton, J. A. (HESS Collaboration) 2004, New Astron. Rev., 48, 331

Hiraga, J. S. 2005, private communication

Hiraga, J. S., Uchiyama, Y., Takahashi, T., \& Aharonian, F. A. 2005, A\&A, 431, 953

Hofmann, W. (HESS Collaboration) 2003, in Proc. 28th ICRC, 2811

Jones, F. C., \& Ellison, D. C. 1991, Space Sci. Rev., 58, 259

Katagiri, H., Enomoto, R., Ksenofontov, L. T., et al. 2005, ApJ, 619, L163

Koyama, K., Petre, R., Gotthelf, E. V., et al. 1995, Nature, 378, 255

Koyama, K., Kinugasa, K., Matsuzaki, K., et al. 1997, PASJ, 49, L7

Lazendic, J. S., Slane, P. O., Gaensler, B. M., et al. 2004, ApJ, 602, 271

Lemoine-Goumard, M., \& de Naurois, M. (HESS Collaboration) 2005, in High Energy Gamma-Ray Astronomy, AIP Conf. Proc., 745, 703

Lucek, S. G., \& Bell, A. R. 2000, MNRAS, 314, 65

Malkov, M. A., \& O'C Drury, L. 2001, Reports of Progress in Physics, 64, 429

Malkov, M. A., Diamond, P. H., \& Sagdeev, R. Z. 2005, ApJ, 624, L37

Mastichiadis, A., \& de Jager, O. C. 1996, A\&A, 311, L5

Moriguchi, Y., Tamura, K., Tawara, Y., et al. 2005, ApJ, 631, 947

Muraishi, H., Tanimori, T., Yanagita, S., et al. 2000, A\&A, 354, L57

Pannuti, T. G., Allen, G. E., Houck, J. C., \& Sturner, S. J. 2003, ApJ, 593, 377

Pfeffermann, E., \& Aschenbach, B. 1996, in Roentgenstrahlung from the Universe, 267

Piron, F., Djannati-Atai, A., Punch, M., et al. 2001, A\&A, 374, 895

Reimer, O., \& Pohl, M. 2002, A\&A, 390, L43

Slane, P., Gaensler, B. M., Dame, T. M., et al. 1999, ApJ, 525, 357

Slane, P., Hughes, J. P., Edgar, R. J., et al. 2001, ApJ, 548, 814

Tsuchiya, K., Enomoto, R., Ksenofontov, L. T., et al. 2004, ApJ, 606, L115

Uchiyama, Y. 2005, private communication

Uchiyama, Y., Takahashi, T., \& Aharonian, F. A. 2002, PASJ, 54, L73

Uchiyama, Y., Aharonian, F. A., \& Takahashi, T. 2003, A\&A, 400, 567

Vincent, P., et al. (HESS Collaboration) 2003, in Proc. 28th ICRC, 2887

Vink, J., \& Laming, J. M. 2003, ApJ, 584, 758

Völk, H. J., Berezhko, E. G., \& Ksenofontov, L. T. 2005, A\&A, 433, 229

Wang, Z. R., Qu, Q.-Y., \& Chen, Y. 1997, A\&A, 318, L59 
1 Max-Planck-Institut für Kernphysik, PO Box 103980, 69029 Heidelberg, Germany e-mail: David.Berge@mpi-hd.mpg.de

2 Yerevan Physics Institute, 2 Alikhanian Brothers St., 375036 Yerevan, Armenia

3 Centre d'Étude Spatiale des Rayonnements, CNRS/UPS, 9 Av. du Colonel Roche, BP 4346, 31029 Toulouse Cedex 4, France

${ }^{4}$ Universität Hamburg, Institut für Experimentalphysik, Luruper Chaussee 149, 22761 Hamburg, Germany

5 Institut für Physik, Humboldt-Universität zu Berlin, Newtonstr. 15, 12489 Berlin, Germany

${ }^{6}$ LUTH, UMR 8102 du CNRS, Observatoire de Paris, Section de Meudon, 92195 Meudon Cedex, France

7 University of Durham, Department of Physics, South Road, Durham DH1 3LE, UK

${ }^{8}$ Laboratoire Leprince-Ringuet, IN2P3/CNRS, École Polytechnique, 91128 Palaiseau, France

9 APC, 11 place Marcelin Berthelot, 75231 Paris Cedex 05, France UMR 7164 (CNRS, Université Paris VII, CEA, Observatoire de Paris), France

10 Dublin Institute for Advanced Studies, 5 Merrion Square, Dublin 2, Ireland
11 Landessternwarte, Königstuhl, 69117 Heidelberg, Germany

12 Laboratoire de Physique Théorique et Astroparticules, IN2P3/CNRS, Université Montpellier II, CC 70, Place Eugène Bataillon, 34095 Montpellier Cedex 5, France

13 Laboratoire d'Astrophysique de Grenoble, INSU/CNRS, Université Joseph Fourier, BP 53, 38041 Grenoble Cedex 9, France

14 DAPNIA/DSM/CEA, CE Saclay, 91191 Gif-sur-Yvette Cedex, France

15 Unit for Space Physics, North-West University, Potchefstroom 2520, South Africa

16 Laboratoire de Physique Nucléaire et de Hautes Énergies, IN2P3/CNRS, Universités Paris VI \& VII, 4 place Jussieu, 75252 Paris Cedex 5, France

17 Institute of Particle and Nuclear Physics, Charles University, V Holesovickach 2, 18000 Prague 8, Czech Republic

18 Institut für Theoretische Physik, Lehrstuhl IV: Weltraum und Astrophysik, Ruhr-Universität Bochum, 44780 Bochum, Germany

19 University of Namibia, Private Bag 13301, Windhoek, Namibia

${ }^{20}$ European Associated Laboratory for Gamma-Ray Astronomy, jointly supported by CNRS and MPG 